\title{
digitalEDUCATION
}

\section{Hacia una visión contemporánea de la Tecnología Educativa}

\author{
Linda Castañeda \\ lindacq@um.es \\ Universidad de Murcia, España \\ Jesús Salinas \\ jesus.salinas@uib.es \\ Universitat de les Illes Balears, España
}

Jordi Adell

jordi@uji.es

Universitat Jaume I, España

\begin{abstract}
Resumen
El objetivo de este trabajo es someter a debate algunos de los temas clave que definen la investigación y la práctica de la Tecnología Educativa académica (TE) en la actualidad y que explican, en parte, su crisis de identidad. El análisis teórico que se presenta centra su atención en cuatro temas: (1) la necesidad de un concepto más actual y matizado sobre qué es la tecnología; (2) una redefinición del campo de estudio de la Tecnología Educativa basada en la relación entre tecnología y educación, ampliando su ámbito de estudio más allá de la dicotomía tradicional entre dispositivos y diseño instruccional; (3) la reconsideración de sus relaciones con otras disciplinas académicas que le sirven como soportes teórico-analíticos; y, finalmente, (4) el análisis de algunas de las debilidades de la investigación actual en TE producto de las asunciones epistemológicas dominantes en las últimas décadas, de las metodologías auspiciadas por las publicaciones y del tipo de conocimiento que promueven.
\end{abstract}

\section{Palabras Clave}

Tecnología Educativa; Diseño instruccional; Filosofía de la tecnología; Nuevas tecnologías aplicadas a la educación; Epistemología 


\title{
Towards a contemporary vision of Educational Technology
}

\author{
Linda Castañeda \\ lindacq@um.es \\ University of Murcia, Spain
}

Jesús Salinas

jesus.salinas@uib.es

Universitat de les Illes Balears, Spain

\section{Jordi Adell}

jordi@uji.es

Universitat Jaume I, Spain

\begin{abstract}
The aim of this paper is to discuss some of the key issues that define the research and practice of academic Educational Technology (EdTech) today and that explain, in part, its identity crisis. The theoretical analysis presented focuses on four themes: (1) the need for a more current and nuanced conceptualization of what technology is; (2) a redefinition of the field of study of Educational Technology, based on the relationship between technology and education, expanding its scope of study beyond the traditional dichotomy between devices/tools and instructional design; (3) the reconsideration of the relations of EdTech with other academic disciplines that serve as theoretical-analytical supports; and, finally, (4) the analysis of some of the weaknesses of current research in EdTech resulting from the dominant epistemological assumptions of recent decades, from the methodologies sponsored by the publications and from the type of knowledge they promote.
\end{abstract}

\section{Keywords}

Educational Technology; Instructional design; Philosophy of technology; New technologies applied to education; Epistemology 


\section{Introducción}

En el campo de la pedagogía, la tecnología educativa (TE en lo sucesivo) ha evolucionado de manera acelerada como ámbito de estudio, investigación y práctica profesional. En los últimos años han crecido de manera exponencial las publicaciones, congresos y jornadas, las asociaciones, fundaciones y comités, los proyectos institucionales de equipamiento de infraestructuras y formación permanente del profesorado, los informes internacionales y el mercado de artefactos y "soluciones" tecnológicas a los problemas de la educación. Esta aceleración es reflejo de la propia evolución de la sociedad: la tecnología es hoy una de fuerzas de cambio más destacadas. Consecuentemente, la TE busca adaptarse a las necesidades del momento y sufre las consecuencias de las fuerzas del cambio. En este tipo de dinámicas, los momentos de estabilidad para elaborar y consolidar ideas son escasos, aunque puede que sean más necesarios que nunca (Biesta et al., 2019).

Parece evidente que en la TE académica existe cierta sensación de que la disciplina necesita reflexionar profundamente sobre sus asunciones epistemológicas, sus objetivos, sus métodos de investigación y de construcción teórica y sus prácticas. Recientemente, por ejemplo, se ha criticado el exceso de propuestas cuantificadoras y experimentales en la investigación (Biesta et al., 2019), la excesiva parcialización de las visiones sobre lo que es y lo que supone la TE (Lai \& Bower, 2019), los problemas derivados de las "guerras de paradigmas" (Jones \& Kennedy, 2011; Kimmons \& Johnstun, 2019) o la evidente falta de fundamento pedagógico en los diseños (Bartolomé et al., 2018; Zawacki-Richter et al., 2019).. Se han hecho llamamientos a visiones más holísticas, críticas, con perspectivas novedosas, que vayan más allá "de lo que funciona" y que exploren también el "cómo funciona", qué es valioso conseguir y qué responde a intereses espurios, los procesos subyacentes y nuevos paradigmas metodológicos (Castañeda, 2019; Castañeda \& Selwyn, 2018; Jameson, 2019; Lai \& Bower, 2019; Williamson et al., 2019). En resumen, diversos autores abogan por visiones más complejas y menos reduccionistas que nos ayuden a comprender mejor las relaciones entre la educación y la tecnología.

La fascinación moderna por la tecnología ha llenado el campo de la TE de promesas, siempre incumplidas (Cuban, 2009; Reiser, 2001). La radio, el cine, la televisión, los ordenadores, la Internet, la inteligencia artificial... la historia de la tecnología educativa parece formada por ciclos de expectativas exageradas, decepción ante los primeros resultados y renovación de la fe ante la aparición de una nueva tecnología que completará todas nuestras expectativas educativas. A la vez, ese ciclo de decepciones refuerza la incertidumbre sobre para qué sirve la $T E$, hasta qué punto sigue siendo necesaria -al menos planteada desde el punto de vista pedagógico- y si hay nuevos caminos por recorrerla.

El objetivo de este trabajo es modesto. Simplemente es "poner encima de la mesa" y someter a debate colectivo algunos temas clave que definen la investigación y la práctica de la TE en el presente y que creemos que explican, al menos en parte, su actual crisis de identidad. La fascinación por las nuevas tecnologías de la información y la comunicación que comenzó a principios de los 90 escondió en buena medida la crisis de identidad del diseño instruccional de los 80 (véase Area (1991) para una síntesis). Hoy, parece que dicha fascinación se ha agotado, al menos en parte de la comunidad académica. En el texto que sigue, en primer lugar, se recoge la idea de la necesidad de proveernos de un concepto más amplio y matizado sobre qué es la tecnología, inspirado en los desarrollos conceptuales de la filosofía de la tecnología de las últimas décadas. En segundo lugar, se trata la relación entre tecnología y educación en el momento presente, es decir, en una sociedad en la que la primera se ha convertido en uno de los motores de trasformación y cambio y a la segunda le son exigidas soluciones más allá de lo razonable; para ello se planteará que es necesario que la TE amplíe su ámbito de estudio superando no solo la dicotomía tradicional de dispositivos y diseño instruccional centrado en el aula, sino el aprendizaje formal en sí mismo y, desde los 90, el ámbito de "las TIC". Para ello se propone una estructura de 
soportes teórico-analíticos que sustentan la TE contemporánea y un diálogo más fluido con otras disciplinas. En tercera instancia se analizan ciertas limitaciones de la investigación en TE, sus asunciones epistemológicas dominantes de las últimas décadas, las metodologías más aceptadas en las publicaciones y el tipo de conocimiento que promueven. Finalizaremos con unas conclusiones -del todo inconcluyentes- que recogen algunas de las ideas expuestas y que pretenden, modestamente, proponer un debate sobre qué es hoy la TE y a qué deberíamos dedicamos quienes decimos que "somos de tecnología educativa".

\section{II.Una definición de tecnología más amplia}

A nuestro juicio, una de las principales fuentes de problemas de la TE es la pobre conceptualización de la tecnología por parte de investigadores y docentes. Algunos autores han señalado que la investigación sobre el valor pedagógico y potencial de la TE se ha visto "limitada por las perspectivas filosóficas implícitas sobre la tecnología que adopta dicha investigación" (Hamilton \& Friesen, 2013, p. 1). Dichas perspectivas constituyen el "sentido común" con el que mucha investigación pedagógica y práctica educativa se acerca a la TE y, de manera generalizada, han respondido a lo largo de la historia a posiciones básicamente esencialistas - convencidas de que la tecnología posee una esencia inmutable y previa a su utilización en contextos concretos-, instrumentalistas -que entienden que la tecnología solo es un instrumento neutral en manos de los docentes al servicio de sus objetivos previos, en muchos casos claramente deterministas (Webster, 2017) y progresivamente empobrecidas (Oliver, 2016).

Oliver (2016) insiste en que la concepción que tenemos sobre la tecnología es determinante a la hora de entender qué hacemos y cómo lo hacemos en TE. Así, "si la tecnología se considera de manera instrumental, el trabajo se orienta hacia cuestiones de eficiencia utilizando un modelo simple y causal; si se considera en términos de las prácticas o la cultura, las cuestiones de significado, experiencia y valor afloran" (Oliver, 2016, p. 42). Un mejor conocimiento de los planteamiento de las distintas corrientes de la filosofía de la tecnología, especialmente tras el "giro empírico" de mitad de los 90 (Brey, 2010), proporcionaría una manera más adecuada de entender la tecnología en general y la educativa en particular.

Como decíamos, entender la tecnología desde una visión instrumental implica limitarse a "una visión centrada en los artefactos, ignorando otros aspectos" (Adell, 2018, p. 119). Pero, ¿a qué "otros" aspectos nos referimos? Cuando hablamos de tecnología deberíamos tener en cuenta que podemos pensarla de cuatro formas diferentes (De Vries, 2012):

a) como una enorme colección de artefactos de los que conocemos no solo sus propiedades estructurales o físicas, sino también sus propiedades funcionales (propia, impropia, cualificadora o técnica).

b) como una disciplina o un dominio de conocimiento, es decir, algo que se puede aprender o estudiar y que es diferente del conocimiento científico. Desde esta perspectiva la tecnología debería ser una disciplina que explora tanto los tipos de tecnologías, como los tipos de conocimiento tecnológico.

c) como una colección de actividades de diseño, elaboración y uso de la tecnología.

d) como un campo de valores humanos y sociales.

Por dicha razón, Oliver (2016) nos invita a una lectura de lo que significa hacer TE desde algunos centros de controversia sobre qué es la tecnología: 
- Tecnología como causa: en esta perspectiva se fundamentan los trabajos relativos a las affordances -tanto a favor como en contra-, la percepción sobre la tecnología y las propiedades intrínsecas de los artefactos tecnológicos.

- Tecnología como intervención social: cuando se estudia si la tecnología favorece, capacita, permite o restringe la actividad social. Suele estudiarse los efectos, la eficiencia, la eficacia o los efectos colaterales de una u otra tecnología.

- Tecnología como efecto social: ¿tiene la sociedad un impacto en el desarrollo de la tecnología? ¿Cómo opera ese impacto en un ámbito concreto?

- Tecnología como ejemplificación o ilustración ${ }^{1}$ de la teoría: cuando se entiende que la tecnología es la forma en la que se da cuerpo, a través del diseño, a la teoría, al mundo de las ideas o viceversa (como en el caso de la investigación basada en el diseño).

- Tecnología como sistema embebido en otros sistemas: cuando entendemos que la tecnología no es una parte más del complejo sistema social, sino que es en sí misma un sistema que funciona dentro de cada uno de los sistemas sociales $y$, por lo tanto, no se presta a explicaciones monolíticas, causales o esencialistas de la realidad, sino a entender casos situados y concretos.

- Tecnología como efecto red: cuando dedicamos nuestra atención a entender la materialidad de la práctica social. Es aquí donde se enmarcan los discursos sociomateriales, incluida las teorías actor-red (actor-network ANT) y los trabajos y desarrollos posteriores.

Desde una perspectiva sociológica y política, Selwyn (2017) entiende la tecnología también como un campo de batalla político e ideológico, Veletsianos y Moe (2017) nos recuerdan la naturaleza sociocultural e ideológica de las tecnologías como fenómeno o Adell (2018) critica los efectos de los mercados en la TE.

\section{III.Tecnología Educativa: la relación entre tecnología y educación.}

La naturaleza misma de la tecnología ha cambiado y nuestra percepción de ella (si es que esto no implica lo primero). Si como hemos planteado antes nuestras concepciones de lo que es la TE han tenido un marcado sesgo instrumentalista, esencialista y/o determinista, todos los análisis clásicos sobre su historia también los tienen. En realidad, remitirnos a la historia de la TE es remitirnos a la historia de la tecnología que impacta instrumentalmente en la educación, o mejor, en el diseño instruccional.

Desde el punto de vista "oficial", la historia de las tecnologías que impactan en la educación contempla básicamente 5 etapas: una primera etapa, previa al origen y desarrollo del concepto (datado por Eraut (1989) en los años 30-40 del S. XX), que podemos considerar las tecnologías ancestrales o la prehistoria de la TE (Cabero, 1996). Entre ellas, merecen especial atención las tecnologías de la imprenta -los libros de texto, por ejemplo-, ya que siguen siendo la tecnología dominante en las aulas y han "moldeado" las prácticas de los docentes desde hace siglos. Después, la historia cuenta hasta cuatro etapas posteriores: la referida a los medios audiovisuales, la centrada en las máquinas de enseñar y los primeros ordenadores, la de las redes telemáticas de comunicación y la de la Web 2.0. A ellas podríamos añadir la siguiente, la derivada de los desarrollos de la inteligencia artificial, que está llamando a la puerta desde hace ya algunos años (Baker et al., 2019), aunque con la preocupante ausencia de los educadores (Zawacki-Richter et al., 2019). Estas etapas no coinciden solo con el desarrollo de un grupo de herramientas

${ }^{1}$ Oliver usa Instantiation que no tiene una traducción literal en castellano. 
concreto, sino con unas formas concretas de entender el papel de dichas herramientas en el proceso educativo. Un resumen de esa evolución lo podemos ver en la figura 1:

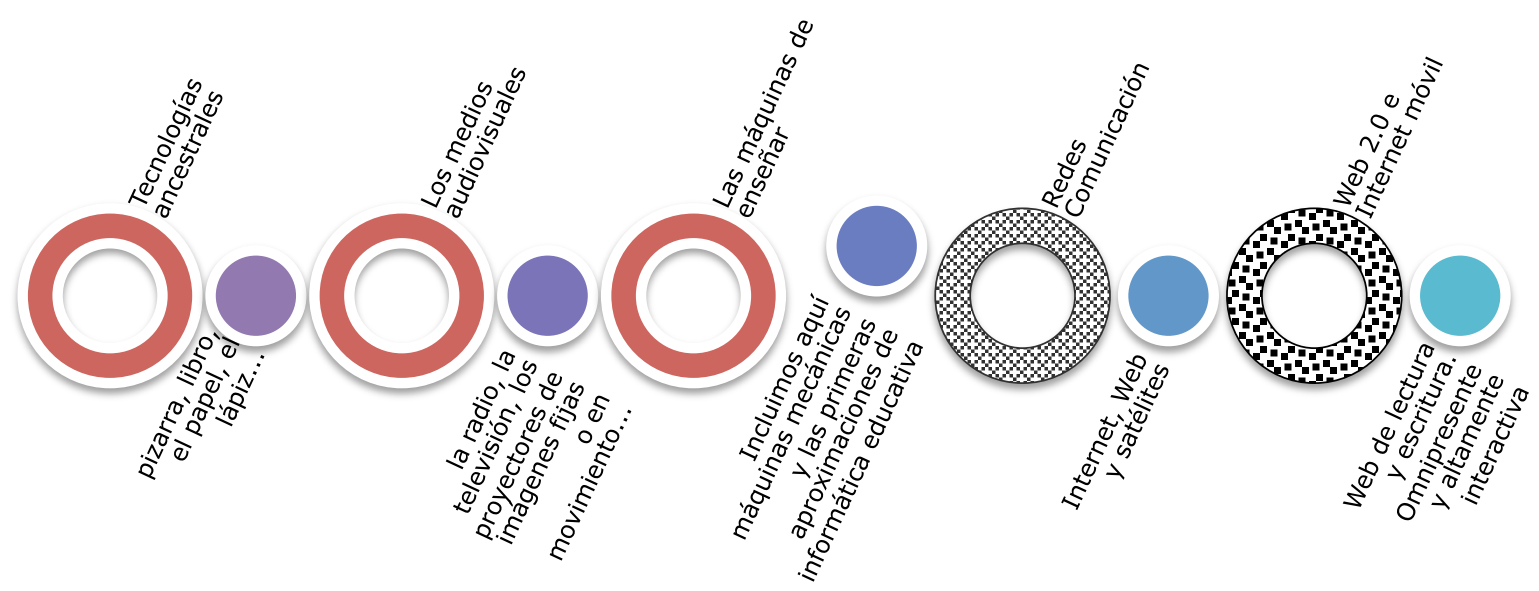

Figura 1: Breve historia de las tecnologías en la educación. Elaboración propia.

Cada uno de estos grupos de tecnologías posee un conjunto de rasgos que le son propios, derivados de su época y de la tecnología dominante, y que afectan de manera radical a las posibilidades, limitaciones, procesos y condiciones de la enseñanza y el aprendizaje en los que pretenden impactar. Así, por ejemplo, la digitalización de la información abrió posibilidades en educación que se han concretado o desvanecido en función de condiciones sociales, económicas y políticas. No hay más que observar los libros de texto digitales actuales (Rodríguez \& MartínezBonafé, 2016) para ver cómo una industria bien establecida y con un mercado cautivo ha concretado la digitalización de sus productos con el objetivo de no perder ingresos invirtiendo lo mínimo imprescindible y reduciendo hasta límites insospechables en su día lo que se puede "hacer" con sus productos.

Hoy, la relación entre tecnología y educación va mucho más allá de la descrita en los estudios evolutivos clásicos y que hemos intentado resumir en la figura anterior (Area, 1991b, 2004; Cabero, 2007; de Pablos, 2009; Sancho, 1994). Ya no son los recursos audiovisuales de los años 40 del siglo $\mathrm{XX}$, desarrollados por el complejo militar estadounidense para la formación en masa de sus tropas, ni son solo el e-learning de la educación a distancia o los MOOC (Liyanagunawardena, Adams, y Williams, 2013; Vázquez-Cano y Meneses, 2015). Tampoco coincide con las máquinas de enseñanza programada de Skinner de los años 50, ni con la Enseñanza Asistida por Ordenador (EAO) de los 70, ni con plataformas de "aprendizaje adaptativo" como Knewton ${ }^{2}$ o Dreambox Learning $^{3}$... ni siquiera eso es suficiente para abarcarlo (Bartolomé et al., 2018). No son tampoco los medios de comunicación de masas, como la televisión educativa o los satélites en los años 80 y 90 (Cabero y Martínez Sánchez, 1995), ni tampoco son solamente los medios de comunicación uno a uno, soportados en redes telemáticas, ni los 2.0, personalizados -al menos en teoría- hasta el

\footnotetext{
${ }^{2}$ https://www.knewton.com/

${ }^{3}$ https://www.dreambox.com/
} 
extremo, omnipresentes en los dispositivos que pueblan los bolsillos -o mejor, las manos- de la inmensa mayoría de la población.

Spector (2016, p. 10) afirma que la TE "implica la aplicación disciplinada del conocimiento con el propósito de mejorar el aprendizaje, la instrucción y/o el desempeño" y, como tal, la caracteriza como una "disciplina de ingeniería" (engineering discipline) que basa su actuación en la teoría, la experiencia y la evidencia científica. Sin embargo, asumir dicho propósito, implicaría perpetuar la visión determinista de la relación entre tecnología y educación (o entre tecnología y aprendizaje), que ya se ha remarcado como uno de los problemas más importantes a la hora de definir y desarrollar el campo de la TE.

Las dicotomías como tecnodeterminismo y sociodeterminismo, incluso en aquellas discusiones sobre una tecnología que "funciona" o no (Clark, 1994; Cuban, 1993; Papert, 1981), son insuficientes a la hora de comprender el efecto de la tecnología en la educación. Aunque el uso de la tecnología en las aulas como soporte cognitivo se ha probado como un elemento de mejora (Tamim, Bernard, Borokhovski, Abrami, y Schmid, 2011), lo cierto es que seguimos sin saber muy bien qué parte exactamente del proceso de implementación de esas tecnologías es el que obra estos efectos.

El papel político que ejercen las tecnologías también en educación (Winner, 1980), la forma en que condicionan nuevas y complejas formas de alfabetización de las personas y configuran modelos culturales y de pensamiento (Martínez Bonafé, 2001), el impacto de las tecnologías digitales en la atención de niños y jóvenes (Hayles, 2007, 2012), los modelos de interacción entre tecnología y sociedad (Bromley, 1997) y entre ciencia, tecnología y sociedad o los múltiples factores relacionados con la agencia de personas y artefactos (Oliver, 2011) nos exigen una mirada mucho más profunda y comprometida sobre la relación entre educación y tecnología.

Dicha mirada implica superar las definiciones consensuadas por la AECT en sus diferentes versiones, tanto de la de 1963, que hablaba de audiovisual communications, como la de 1977, que hablaba ya de Educational Technology y que incluía no solo el impacto instrumental de la tecnología en la instrucción formal, sino en la organización escolar y en el aprendizaje informal. También la de 1994, en la que se restringe el campo a la Instructional Technology para intentar "acotar" el ámbito de estudio o la de 2004, que, aunque vuelve a abrir la perspectiva a la Educational Technology e incluye aspectos éticos, sigue centrándose en el aprendizaje y la instrucción y la mejora de la performance 4 con el aprovechamiento instrumental de la tecnología (AECT Definition and Terminology Committee, 2004; AECT Task Force on Definition and Terminology, 1979; Januszewski, 2001; Saettler, 1978, 1990). Incluso la de 2008, que incluye no solo recursos tecnológicos, sino también procesos tecnológicos (Ibrahim, 2015), se queda corta. Tampoco podemos quedarnos en planteamientos actuales que la reduzcan a una mera "teoría del aprendizaje mediado por tecnología" (Bower, 2019).

No podemos ignorar la importancia de las definiciones anteriores. Como bien apunta Januszewski (2001), cada definición es hija de su tiempo y de su contexto y, lo que es más importante, tiene una finalidad en él. Vivimos tiempos en los que ver a las tecnologías de forma crítica en el marco de las sociedades entendidas de manera compleja ya no es una labor propia de apocalípticos, sino que es una exigencia de responsabilidad frente al futuro (Castañeda y Selwyn, 2018). La cuestión es que la tecnología ni incide en la educación solo cuando es "nueva", ni se "aplica" a la

\footnotetext{
4 "Educational technology is the study and ethical practice of facilitating learning and improving performance by creating, using, and managing appropriate technological processes and resources" (AECT Definition and Terminology Committee, 2004, p. 3)
} 
educación ${ }^{5}$, ni se "implementa" en ella, ni la "mejora" (enhanced) per se, todo esto eso es solo una parte de lo que la tecnología puede hacer en educación. De la misma manera que la política, la antropología, la psicología o la sociología condicionan la educación y todos los aspectos vinculados con ella (dentro y fuera de ámbito formal), la tecnología, además de ser instrumento dentro del llamado diseño instruccional (su clásico objeto de interés en el marco de la didáctica (Bartolomé, 2008), y un condicionante sistémico de la educación como proceso especializado de comunicación (Martínez Sánchez y Prendes, 2003; Martínez Sánchez, 2007; Prendes, 1997)), también explica y redimensiona cada uno de los aspectos educativos de forma más amplia y profunda de lo que hemos pretendido. Por ello, ubicar a la tecnología como un apéndice de cualquier otro proceso incluida la educación- resulta, a nuestro juicio, reduccionista y problemático y deja muchos de los aspectos de la realidad de ese proceso (el que sea) casi "desprotegidos" ante alguna de las "naturalezas" de la tecnología.

La educación es con tecnología, porque la sociedad es con tecnología en el más completo sentido de lo posdigital (de Laat \& Dohn, 2019; Taffel, 2016) y obviar alguno de los aspectos de la educación en la reflexión sobre la tecnología nos lleva a generar discursos y prácticas parciales, a descontextualizar todos los procesos educativos y a ignorar -casi ingenuamente- la influencia de la tecnología en ellos. Nos creemos inmunes a los cambios, pero la educación no puede ignorar que los seres humanos somos también producto de la tecnología de la época que nos ha tocado vivir (Hayles, 2016; Stiegler, 2015). Todos y cada uno de los pilares básicos de la educación afectan a la tecnología educativa, de la misma manera que la tecnología educativa impacta en todos los aspectos de la pedagogía: la tecnología les afecta, condiciona y amplifica.

En consecuencia, la tecnología educativa necesita una definición que aluda a esa mirada más profunda y comprometida, una definición que pase por caracterizarla de forma general como un campo de conocimiento que formula preguntas y busca respuestas de índole educativa acerca de la compleja relación entre las personas y la tecnología en todos los ámbitos de la educación.

\section{IV.Soportes teórico-analíticos e interlocutores de la tecnología educativa}

Plantear una reformulación de la TE que incluya cómo se relacionan tecnología y educación en su sentido más amplio, implica una reflexión profunda sobre algunos de los planteamientos más clásicos, recurrentes y, por ende, básicos de la tecnología educativa, por ejemplo, el referido a sus bases conceptuales.

Como mencionan todos los trabajos al respecto y sintetiza Ely (2008), las bases de lo que llamaremos TE "clásica" se sustentan en los tres pilares básicos ya mencionados en su momento por Chadwick:

- La psicología.

- Las teorías de la comunicación.

- La teoría general de sistemas.

Sobre estos tres pilares básicos, importados de la tradición anglosajona y que poco tenía que ver

\footnotetext{
${ }^{5}$ Alusión a la denominación de la asignatura de los planes de estudio de las primeras asignaturas de Tecnología Educativa en los planes educativos de formación de maestros en las universidades españolas (año 1997):" Nuevas Tecnologías Aplicadas a la Educación".
} 
con nuestra tradición pedagógica más "europea continental", se ha asentado no solo el conocimiento propio del campo, sino la práctica habitual de la profesión. A estos tres pilares, al menos en el contexto nacional, se suele sumar la didáctica como marco contenedor (no en vano en esa visión clásica la TE se circunscribe a la instrucción como una parte del proceso educativo).

Esta visión clásica de los tres pilares -por otra parte, muy propia del contexto anglosajón-, y probablemente como consecuencia del desarrollo de la teoría crítica, desde mediados de los años 90 del pasado siglo, se vio cuestionada hasta cierto punto por visiones alternativas que reclamaron perspectivas más críticas, holísticas y socialmente comprometidas de la TE. En tales alternativas, se sugería que se aprovechase la influencia de otros ámbitos como la semiótica, la sociología de la educación (p.e., Prendes, 1997). Sin embargo, como bien sintetiza la misma autora, en algunos casos esos planteamientos se consideraron demasiado generalistas e incluso "desnaturalizadores" del campo de estudio, siendo prácticamente ignorados en los desarrollos posteriores. Otros desarrollos, si bien ampliaron la visión de los ámbitos que ejercían influencia en la TE, seguían concentrando el efecto en el proceso de comunicación.

Hay que decir que los momentos tecnológicos en los que se plantearon aquellas visiones alternativas, estuvieron marcados por las repercusiones sociales más evidentes de los cambios "del mundo conectado" -no olvidemos que la década de 1995-2005 es la del desarrollo y popularización de la Web y la de 2005-2015 la de la llamada Web 2.0 y los dispositivos móviles- y por un casi inevitable optimismo asociado a ellos (la Web de lectura y escritura, la democratización de los contenidos, los prosumers, etc.), que empujó -azuzó incluso- el devenir teórico práctico de la TE y convirtió en inoportunos o extemporáneos -al menos eso parece desde la perspectiva actual-, todos los intentos de cambio sustancial o profundo en la disciplina.

Sin embargo, a estas alturas del análisis y en el momento de desarrollo tecnológico y social actual, es posible que nos encontremos en un punto donde la propia evolución de la TE haga inevitable que al fundamentarla ampliemos, y hasta cierto punto cambiemos, el foco de atención hacia una concepción más pedagógica.

Proponemos que no solo hablemos de psicología de la educación y teorías del aprendizaje, sino que hablemos con propiedad de teorías de la enseñanza desde la pedagogía; que no hablemos de la didáctica como contenedora, sino como interlocutora; que no hablemos de la teoría de sistemas desde la perspectiva gerencialista de la provisión de medios, sino desde la perspectiva social y de la organización de las instituciones educativas; que veamos que la sociología de la educación y la teoría de la educación no se pueden considerar pequeños pilares, sino pilares de dimensiones considerables en un contexto tecnológico y social donde, de no hacerlo, se corre el peligro de caer en la más absoluta irrelevancia.

A continuación se plantea una brevísima revisión de las bases que consideramos contribuyentes e interlocutores inexcusables de una TE actual, empezando por revisar los clásicos, su especial contribución al campo y proponemos, aunque sea de forma breve, otros campos con los que entendemos que en la actualidad la TE debe mantener una relación de mutua influencia y diálogo, si es que quiere tener una vocación más holística que abarque comprensivamente a toda la educación.

\section{a. TE y Filosofía de la Tecnología}

No es baladí empezar este repaso remarcando la importancia de observar cuidadosamente los desarrollos en la filosofía de la tecnología. En este caso no se trata de una disciplina con la qué mantener un diálogo desde la $T E$, sino que hay que considerarla uno de sus puntos de anclaje. Como dijimos ya en uno de los primeros apartados de este trabajo, es evidente que en la medida en que podamos mantener perspectivas actuales sobre qué es la tecnología (o, mejor, las 
tecnologías) y seamos capaces de traducirlas en lo que hacemos, tendremos miradas más abiertas de nuestro campo de estudio.

Pero ¿qué aporta la filosofía de la tecnología a la TE? La filosofía de la tecnología, especialmente tras su "giro empírico" (Achterhuis, 2001; Brey, 2010), nos ofrece visiones tan sugerentes para la educación como su papel mediador en nuestra relación con el mundo y metodologías de análisis (posfenomenología), su carácter netamente político (teoría crítica de la tecnología) o su carácter antropogenético e individualizador, de pharmakon (veneno y cura) y sobre los mecanismos del sistema tecno-industrial contemporáneo (Stiegler), por citar algunos solo algunos autores. Debería interesarnos no solo el "impacto" educativo de las tecnologías concretas en personas concretas y en la sociedad en general, sino los procesos que subyacen a su diseño, difusión, adopción y uso, los supuestos epistemológicos y pedagógicos en las que se basa su diseño y uso, sus efectos pretendidos (eficacia y eficiencia), pero también los no pretendidos $y$, en general, cómo la tecnología da forma a los procesos educativos formales, informales y no formales, incluso a su moralidad (Kroes \& Verbeek, 2014), en una época en la que, por ejemplo, algoritmos opacos están tomando decisiones pedagógicas (Beer, 2017; P. B. de Laat, 2017; Williamson, 2017).

Reflexiones recientes como las de Czerniewicz (2008), De Vries (2012), Hamilton y Friesen, (2013), Oliver (2016), Selwyn (2017), Webster (2017) o Feenberg (2017), entre otros, nos han ayudado a comprender la necesidad de que la TE parta de una comprensión más amplia de qué es la tecnología y cómo está cambiando el mundo y a nosotros mismos.

\section{b. TE y teoría de la complejidad}

Siguiendo a Ely (2008), la llegada de la teoría de sistemas a la TE "estimuló una gran cantidad de modelos de desarrollo instruccional que han sido repetidamente usados por los prácticos del campo" (Ely, 2008, p. 247). No obstante, dichos modelos trajeron también al campo de la TE el gerencialismo, y se convirtieron, por diversos motivos, en una sucesión de modelos de "solución" para la gestión de sistemas de información, análisis de coste-eficiencia o evaluación de personal. Hoy, seguramente, el paso obvio en TE es superar la visión sistémica de la influencia de la tecnología y apostar por una visión basada en la Teoría de la Complejidad, que como bien apuntan Fenwick, Edwards y Sawchuk, resulta más comprensiva a la hora de explicar las actuales condiciones mundiales de la educación, teniendo en cuenta "la interconexión en constante evolución de las ideas, los procesos y las organizaciones, y la enredada proliferación de redes mediadas por la tecnología" (2011, p. 50).

La teoría de la complejidad no es un cuerpo monolítico de ideas, sino más bien un conjunto de desarrollos que incluyen a la teoría general de sistemas, la cibernética, la teoría del caos, autopoiesis y la ecología profunda y que puede definirse como "la comprensión interdisciplinar de la realidad como compuesta de sistemas abiertos complejos con propiedades emergentes y potencial transformador" (Byrne, 2005, p. 97). Eso quiere decir que no solo entendemos la realidad donde se enmarca la $T E$, la educación, como un sistema, sino como un conjunto de sistemas complejos, en los que sus componentes tienen agencia, y abiertos, en los que fenómenos, eventos y actores (humanos y no humanos) son mutuamente dependientes y de su relación afloran (emergen) estructuras nuevas.

La influencia de la teoría de la complejidad se ha dejado sentir con fuerza en el ámbito de la TE, no solo en la generación de movimientos de investigación novedosos, cada vez más presentes en el panorama de la investigación mundial (la Teoría de la Complejidad está en la base del paradigma sociomaterial), sino como alternativa y revulsivo a las visiones anteriores. 


\section{c. TE y la organización de las instituciones educativas y no educativas}

Cuando hablábamos de la teoría general de sistemas como base de la TE aludimos al impacto del gerencialismo en la TE. Como decíamos más arriba, los tecnólogos educativos -u otros profesionales- hemos tomado decisiones basados en modelos gerencialistas de provisión de recursos que, en su mayoría, no entendíamos en profundidad (Ely, 2008). Esas decisiones no solo se toman en instituciones educativas, sino también en instituciones de todo tipo, donde trabajan o se relacionan personas y se generan situaciones de aprendizaje.

Por eso mismo, hoy sabemos que la TE tiene implicaciones organizativas en la dinámica y estructura de las instituciones educativas y que, a la vez, las dinámicas y estructura de estas instituciones educativas tienen un impacto trascendental en la TE. Un ejemplo de su importancia es el Marco Europeo para Organizaciones Educativas Digitalmente Competentes (European Union, 2015a, 2015b) o las reflexiones sobre las dinámicas organizativas a la luz del momento tecnológico actual de Starkey (2012). También la organización de instituciones no educativas en procesos de aprendizaje permanente dialoga activamente con la TE, como por ejemplo las propuestas como las de los Entornos Organizativos de Aprendizaje, conocidos por su acrónimo en inglés OLE (Organizational Learning Environments) (Parejo y Olmedo, 2017).

\section{d. TE y la Didáctica}

Entendida de manera clásica como el campo disciplinar que se ocupa de los procesos de enseñanza-aprendizaje, la didáctica es caracterizada por (Sarramona \& Fernández, 1978) como una de las ciencias aplicativas dentro de las llamadas Ciencias de la Educación. En ella confluyen campos como la enseñanza general y especial, el currículum, las técnicas de instrucción, la formación del profesorado, etc.

Este es el marco en el que la TE clásica se ha ubicado en nuestro país: el estudio aplicado de cómo la tecnología se usa en los procesos de enseñanza-aprendizaje, en los procesos de instrucción, y por lo tanto en el marco del diseño instruccional, objeto directo de la didáctica (Bartolomé, 1988; Cabero, 2007; Prendes, 1997). Sin embargo, ya Salinas en su momento afirmó que "ni la TE agota el campo de la Didáctica, ni queda encerrada entre sus límites. La Didáctica abarca aspectos a los que no llega la TE y, ésta, se ocupa de campos que son externos a la Didáctica, es decir, no estrictamente instruccionales" (Salinas, 1991, p. 24). La Didáctica aporta a la TE fundamentalmente, dos cosas: "modelos del proceso enseñanza-aprendizaje desarrollados a partir de la teoría y modelos de investigación aplicada" (Salinas, 1991, p. 32). Y, efectivamente, la relación didáctica-tecnología no se agota en un marco, sino que se define mejor en una relación de mutuo diálogo e influencia.

Para poder entender cómo la tecnología impacta en los procesos educativos, y para hacer un análisis realmente holístico de ese impacto (reactivo, proactivo o meramente analítico) es imprescindible conocer el modelo curricular en que nos enmarcamos, y cómo cada uno de los condicionantes de esa teoría, mejor, discurso curricular (sea entendido desde la perspectiva que sea), se define e interactúa en él (Da Silva, 1999).

A la vez, para poder entender de forma adecuada cómo son y cómo se materializan los métodos de enseñanza, la formación del profesorado, los contenidos o las metas educativas hoy, es imprescindible que la Didáctica mire con atención y dialogue fluidamente con la TE (Williamson, 2013b). Ya no es solo una cuestión instrumental. Sin ir más lejos, todos los aspectos de la profesión docente (Castañeda et al., 2018; Esteve et al., 2018), se ven afectados de forma directa e intensa por la tecnología entendida como actividad, como valores, como contexto, como instrumento (sírvanos como ejemplo el análisis de Martínez Bonafé, 2001 sobre cómo afecta la tecnología al compromiso social de la escuela), y en todos ellos el diálogo mutuo TE-didáctica debe 
ser evidente.

\section{e. TE y la psicología del aprendizaje}

La psicología ha tenido una muy marcada influencia en la TE desde el principio de su conceptualización y durante todo su desarrollo. Tanto las teorías del aprendizaje, como las teorías sobre la configuración misma del pensamiento humano han tenido una trascendencia notable en la TE.

Esa influencia es muy evidente cuando vemos como en los tiempos en los que la TE se focalizó en los medios de comunicación de masas fue la psicología de la percepción la que dio cuerpo a los principios de diseño y uso de los materiales. La psicología conductista está en la base de la enseñanza programada, las máquinas de enseñar y, aún hoy, en gran parte de los que se ha venido en llamar "aprendizaje adaptativo" (Bartolomé et al., 2018) y teorías cognitivas, como las de Sweller o Mayer, en los marcos de cuestiones de plena actualidad como el diseño de videojuegos didácticos (Mayer, 2019). La entrada del constructivismo, del constructivismo social, aspectos relativos a la tutorización de los estudiantes (como los incluidos en la zona de desarrollo próximo de Vygotsky o la teoría del andamiaje de Bruner), ampliaron la mirada a nuevos enfoques también en TE (Czerniewicz, 2010).

Según los historiadores de la TE de tradición anglófona, después de la psicología del aprendizaje, pasamos a hablar de psicología de la enseñanza (Ely, 2008). Sin embargo, este es otro camino que concierne a la pedagogía y, muy en particular, a la didáctica. No obstante, no les podemos achacar nada a esos historiadores, máxime teniendo en cuenta que la pedagogía es algo prácticamente inexistente en la tradición anglosajona. Esta cuestión, las diferencias entre las tradiciones anglosajona del currículum y continental de la Bildung, ha sido y sigue siendo la causa de incompresiones y desencuentros entre los investigadores de ambas tradiciones. Su impacto en la TE de origen norteamericano que ha dominado el campo durante décadas merece más espacio de la que podemos dedicar aquí (Biesta, 2011).

Sin embargo, es preciso llamar la atención sobre aportaciones actuales de la psicología que tienen un altísimo impacto cuando hablamos de TE. Por ejemplo, habría que conocer y asumir de forma más consciente algunos desarrollos de la psicología sobre la manera en la que deberíamos entender los aprendizajes en la era digital. Por ejemplo, la necesidad de contribuir al entrenamiento de la metacognición y la autorregulación como funciones cognitivas de interés prioritario en el mundo tecnológico que nos rodea (Azevedo, 2009; Dabbagh y Kitsantas, 2012) o a los principios de diseño de procesos de aprendizaje que contribuyen al aprendizaje personal (que no personalizado) o la importancia del aprendizaje personalizado en el sentido que dan autores como (Coll, 2016) al término.

Es más, desde la psicología se han propuesto en los últimos tiempos ideas más radicales, que han cuestionado algunas de las nociones tradicionales sobre dónde o cómo tiene lugar aprendizaje de las personas en estos tiempos de tecnología. No solo nos referimos a los postulados más audaces del constructivismo social en tiempos de redes, el aprendizaje interconectado (desarrollo de aquel tímido conectivismo) y la noción de que el conocimiento se mueve entre nodos de la red; sino de la llamada psicología Cyborg, en la que se entiende que no solo la persona y la tecnología se influyen sucesiva y mutuamente en un continuo de aprendizaje, sino que el "responsable" del aprendizaje, de los aciertos y de los errores, ha dejado de ser el cerebro desnudo. Parafraseando a Clark (2003, p. 26) diríamos que el verdadero motor de resolución de problemas queda constituido por una matriz biotecnológica más grande que comprende el cerebro, los papeles apilados, los errores anteriores, los archivos electrónicos, las operaciones de búsqueda proporcionadas por el software que usamos en la máquina que usamos, etc., y así sucesivamente. Y como continúa Clark, "lo que 
mejor sabe hacer el cerebro humano es aprender a trabajar en equipo en un campo de resolución de problemas poblado por una increíble variedad de accesorios, andamios, instrumentos y recursos no biológicos. De esta manera, los nuestros son esencialmente los cerebros de los ciborgs nacidos en la naturaleza".

\section{f. TE y teoría de la comunicación}

La teoría de la comunicación es, quizá, la más conocida, persistente e importante teoría en uso en la TE en los últimos años. Desde el momento en que el modelo de Shannon y Weaver (1949) irrumpe en el panorama de la educación y genera, junto a toda la pléyade de teorías posteriores, un marco en el que entendemos a la educación como un proceso de comunicación especializado en el que las tecnologías actúan como mediadoras de ese proceso de interacción, la TE no volvió a ser la misma.

Setenta años después de Shannon y Weaver sabemos que las tecnologías no actúan solo como medios o canales en ese proceso y que las "entidades" que interactúan funcionan de forma bastante más compleja (Floridi, 2016, 2017). El interaccionismo simbólico, derivado de la Escuela de Chicago y los estudios de G.H. Mead nos mostró, por ejemplo, que las personas nos relacionamos con otras personas y con los objetos en base a los significados que les atribuimos, que el lenguaje es una herramienta de negociación de significados y que interpretamos los símbolos (Blumer, 1962). Y esa caracterización del contexto del interaccionismo simbólico, definido por sus rasgos estáticos y emergentes, que es construido y reconstruido constantemente por los participantes durante sus interacciones simbólicas, sigue informando algunos de los principales enfoques de investigación cualitativa.

Las tecnologías no solo "facilitan" los procesos de comunicación que están en la base de la educación, sino que median y condicionan la misma naturaleza de la entidad que se relaciona. Incluso presumimos que ese impacto de la tecnología educativa afecta a los procesos de comunicación dentro y fuera de la escuela. Hoy sabemos que las tecnologías de la información y la comunicación no son solo medios de comunicación. Sabemos también que son herramientas de poder y de influencia, de reconfiguración de la realidad y de reconfiguración colectiva de la misma organización mental que nos permite relacionarnos. La Escuela de Frankfurt nos hizo conscientes de que las tecnologías de la comunicación de masas facilitan la creación de productos culturales básicos de entretenimiento y su consumo tiene un propósito ideológico y sentó las bases de la tecnología educativa critica (Nichols \& Allen-Brown, 1996).

La comunicación es una de las bases fundamentales de la educación, pero la comunicación que no puede reducirse al envío de mensajes por un canal entre un emisor y un receptor. La tecnología condiciona y "media" la comunicación, por tanto, la tecnología educativa precisa de las teorías de la comunicación.

\section{g. TE- Informática y desarrollos tecnológicos posteriores}

Como hemos dicho ya, el mundo actual no es entendible sin comprender el impacto en su configuración y desarrollo de la computación, la informática y las redes telemáticas. Entender la sociedad sin Tecnologías de la Información y la Comunicación (TIC) es prácticamente imposible, pensar en educación, las formas de pensar en cómo aprender, sin tener en cuenta la computación, las TIC, y la Web 2.0 es plantear una educación para mundos que rozan la ficción (Adell, 2010; Cabero, 2007; Cuban, 1993; Martínez y Prendes, 2003; Papert, 1981).

Por ejemplo, hoy al hablar de TE tenemos que hablar de las TIC que influyen en la educación, pero a la vez la TE influye en el desarrollo de esa informática; así si las TIC -en tanto que tecnologías de 
la comunicación- pueblan los procesos comunicativos de las personas que aprenden a lo largo de toda su vida, sus desarrolladores prestan atención a lo que ese lifelong learning requiere (Attwell, 2019). Máxime, cuando la educación en ese sentido ubicuo y permanente se ha convertido, de la mano de los recursos tecnológicos, en un mercado potencial con unas cifras de record, aún por explorar.

No obstante, además de esa visión informática (telemática para ser más precisa) de la TE que es ineludible, si algo es cada vez más obvio, es que la utilización de las inmensas masas de datos que producen las tecnologías es, en sí misma, una de las tecnologías que más efecto pueden tener en la realidad social. Cada uno de los sensores de la llamada Internet de las cosas recoge las huellas dejadas por todo lo que pasa en nuestro mundo de eventos. Ahora no es preciso tomar una muestra de eventos para "presumir" una realidad, podríamos -en algunos casos- tener acceso a la totalidad de ellos y, gracias a la velocidad de procesamiento que conseguimos, convertir esa inmensa cantidad de información en conocimiento a través de inferencias más o menos atrevidas sobre el impacto de esos eventos en otras parcelas de la vida de las personas como el control de recursos en una ciudad -tráfico, seguridad, bomberos, etc.-(Provost y Fawcett, 2013; Waller y Fawcett, 2013), la huella de personas o eventos en un ecosistema concreto, cronobiología o ese Quantified self de que venimos hablando desde hace ya algunos años (Swan, 2013; Whitson, 2013), entre muchos otros.

Y, aunque a primera vista pudiera dar la impresión de que seguimos hablando de informática, esa utilización no tiene que ver tanto con la informática o la computación (aunque tenga que agradecer mucho a ambas), sino con ese término paraguas que conocemos como "inteligencia artificial" y que describe diversas tecnologías y métodos, como el aprendizaje automático, el procesamiento del lenguaje natural, la minería de datos, las redes neuronales o el uso de ciertos algoritmos para que los ordenadores puedan realizar "...tareas cognitivas, generalmente asociadas con las mentes humanas" (Baker et al., 2019, p. 10). O incluso podríamos decir que tiene que ver más exactamente con eso que ha dado en llamarse la "Ciencia de los Datos" (Data Science), (Dhar, 2013).

La aplicación de esa ciencia de datos a los procesos educativos es lo que se ha llamado learning analytics, y por sus características se trata de un campo que puede tener unas tremendas aplicaciones e implicaciones en la educación, en tanto que proceso, en tanto que sistema, en tanto que derecho (Clow, 2012). Y, como bien dice Clow, no se trata solo de entender qué datos tenemos para ver qué pedagogía aplicamos a esos datos (una de las visiones más naif que hacemos en la actualidad de las analíticas del aprendizaje), sino de que todas las partes del ciclo tienen profundas implicaciones educativas que deben explicitarse y entenderse para poder tomar decisiones sobre ellas.

Tal es la importancia de su relación con la educación, que si uno de los pilares de la educación es la alfabetización de las personas y la llegada de las TIC supuso enormes retos en lo que a la alfabetización se refiere (Area, Gros, y Marzal, 2008; Buckingham, 2006; Ferres, 2014; Glister, Rlister, y Gilster, 1998), hoy hablamos también de la alfabetización en datos como clave, tanto para los ciudadanos, como para los educadores (Carlson \& Johnston, 2015; Knight et al., 2017; Pangrazio \& Selwyn, 2018).

\section{h. TE y Política Educativa}

Una de las relaciones que más ha evolucionado en los últimos tiempos entre la TE y otras Ciencias de la Educación es, a nuestro juicio, la relación con la Política Educativa.

Gracias a la investigación sobre el impacto de la política en la educación, sabemos que para que las políticas se conviertan en prácticas de aula deben aprobar una serie de procesos complejos de 
materialización (muchos más difíciles que los primeros acercamientos al enfoque clásico de adopción de políticas) (Mintrom \& Vergari, 1998). Esos procesos se ajustan a caminos retorcidos que marcan muchas diferencias y transformaciones en su ruta hasta el aula (S. Ball, 2013; Caena, 2013; Colebatch, 2015).

Estas transformaciones afectan la política propuesta, así como la práctica en la que se pretende implementar (lo que supone los dos impactos obvios), pero también afecta a los individuos, comunidades e instituciones involucradas en el proceso (Ball, 2016; Ball, Maguire, Braun, Perryman, y Hoskins, 2011; Honan, 2010). Los participantes de la ruta, junto con los marcos que de le dan contexto, afectan cada parte del proceso de transformación de la política. La materialización de una política determinada en la realidad escolar, se convierte en un proceso complejo y profundamente transformador (Ball, 2016; Thomson, Hall, y Jones, 2010).

Además, es crucial la influencia de otros actores en los procesos de toma de decisiones políticas (McCann, 2011). Los políticos y las instituciones oficiales son solo una pequeña parte de las personas que influyen en la toma de decisiones de las políticas de educación pública (Spring, 2012). Muchas redes o comunidades de actores, fuera del gobierno, juegan un papel importante. A pesar de su falta de legitimidad, se ven reforzados como actores principales, ya que representan a una amplia gama de partes interesadas, y contarlo (o venderlo) se entiende como una manera de aumentar la democracia participativa en la toma de decisiones políticas (Menashy, 2015; Stone, 2008).

Pero el impacto de estos actores (los no oficiales), no solo es crucial en el nivel de toma de decisiones, sino también en la difusión, traducción y promulgación de estas políticas (S. Ball, 2013). Como muchos autores afirman (Granovetter, 1983; Mintrom \& Vergari, 1998; Rogers, 2003), el impacto de las personas en la difusión de innovaciones es algo sumamente importante. El papel de la red y de cómo las personas difunden y transforman los discursos políticos y políticotecnológicos en la Web podría cambiar completamente la forma en que una política específica se disemina, traduce y materializa, o impone, resiste o no se comprende (Ball, 2013, 2016; Menashy, 2015; Mintrom y Vergari, 1998).

\section{i. TE y Sociología de la educación}

La llegada de lo digital a la escuela se realizó con muchas promesas: aumentar la igualdad, enfoque social, pensamiento crítico, democratización del acceso, colaboración y muchas otras (Cobo, 2016). Muchas promesas no se cumplieron, por el contrario, muchos problemas (democratización, pensamiento crítico, entre otras), en lugar de mejorar, han empeorado (Selwyn, 2016a, 2016b).

En base a esas promesas rotas, hay muchas realidades que aluden a la sociología de la educación sobre las qué reflexionar.

Por un lado, la aparición de otros actores sociales, proveniente desde fuera de los sectores educativos tradicionales (fundaciones, empresas y muchos otros), en la creación del discurso de la tecnología educativa (análogos a los que nos referimos cuando hablamos de la relación con la política educativa) ha sido señalada como uno de los mayores problemas (Watters, 2016). Además, la creación del gran mercado en torno a la tecnología y que mueve miles de millones de euros (Buckingham, Scanlon, y Sefton-Green, 2001; Selwyn, 2016a; Watters, 2016) se destaca como un problema que dificulta diferenciar las prioridades educativas de las preocupaciones del mercado. Finalmente, las compañías y los vendedores están cambiando los entornos profesionales de los profesores de igual a igual (por ejemplo, conferencias), en espacios para la autopromoción de profesores en celebridades de EdTech (Honan, 2010; Player-Koro et al., 2017).

Pero no solo hay un problema contextual. El enfoque simplista de la tecnología educativa está lleno 
de palabras comerciales (palabras aprendidas durante los tiempos digitales como personalizado y adaptativo, entre otras) que evitan una profunda reflexión pedagógica a favor de otros enfoques más ligeros y es percibido como una realidad y un problema. (Castañeda, 2016; Cobo, 2016). Este enfoque superficial promueve, al menos según la visión de los autores, no solo la falta de reflexión, sino la implementación acrítica de pedagogías vestidas en esos términos modernos, implicando principios tradicionales, si no reaccionarios (es decir, aprendizaje adaptativo) (Selwyn, 2016a; Watters, 2016; Williamson, 2015).

El diálogo TE-SE (Sociología de la Educación) es inevitable si queremos superar esas visiones.

\section{j. TE y otras ciencias de la educación}

Existen otras ciencias de la educación con las que es imprescindible que la TE mantenga un diálogo abierto, explícito y constante. De entre ellas, querríamos destacar su íntima relación con la Economía de la Educación y, muy especialmente, con la Teoría de la Educación.

$\mathrm{Si}$ algo se destaca en las revisiones a las que aludíamos en los primeros apartados de este documento es la escasez -cuando no completa elusión- no solo de el respaldo de teorías en la construcción de investigación en tecnología educativa (Bartolomé et al., 2018), sino de la escasa generación de teoría que debata, defienda, confirme, renueve o desarrolle los fundamentos sobre los cuales seguir construyendo. No parece un mal nuevo, ni siquiera uno exclusivo de la TE, sino extensible a las ciencias de la Educación en general (Ball, 1995) e incluso a las ciencias sociales. Como bien apuntan Costa, Hammond y Younie (2019), la teoría de la educación, y su relación con la $\mathrm{TE}$, en concreto la teoría en la tecnología educativa, debería ayudarnos no solo a aportarnos lentes sobre los fenómenos relacionados con tecnología y educación, sino a proporcionarnos un lenguaje y un vocabulario para desarrollar la comprensión y la crítica. En palabra de estos mismos autores, "la teoría es importante ya que desarrolla una nueva capa de lenguaje que es capaz de iluminar, a veces amplificar, la comprensión del mundo que pretendemos explorar"(Costa et al., 2019 , p. 396) y eso nos conmina a fortalecer y desarrollar debidamente esa relación entre Teoría de la Educación y TE.

Insistimos en que no se trata de una relación unidireccional, como se aprecia en la figura 2 , sino de un diálogo que permita entender tanto en los niveles micro, como en los niveles macro, cómo se incluyen mutuamente y cómo ambas en interacción tienen una influencia determinante en la realidad educativa. 


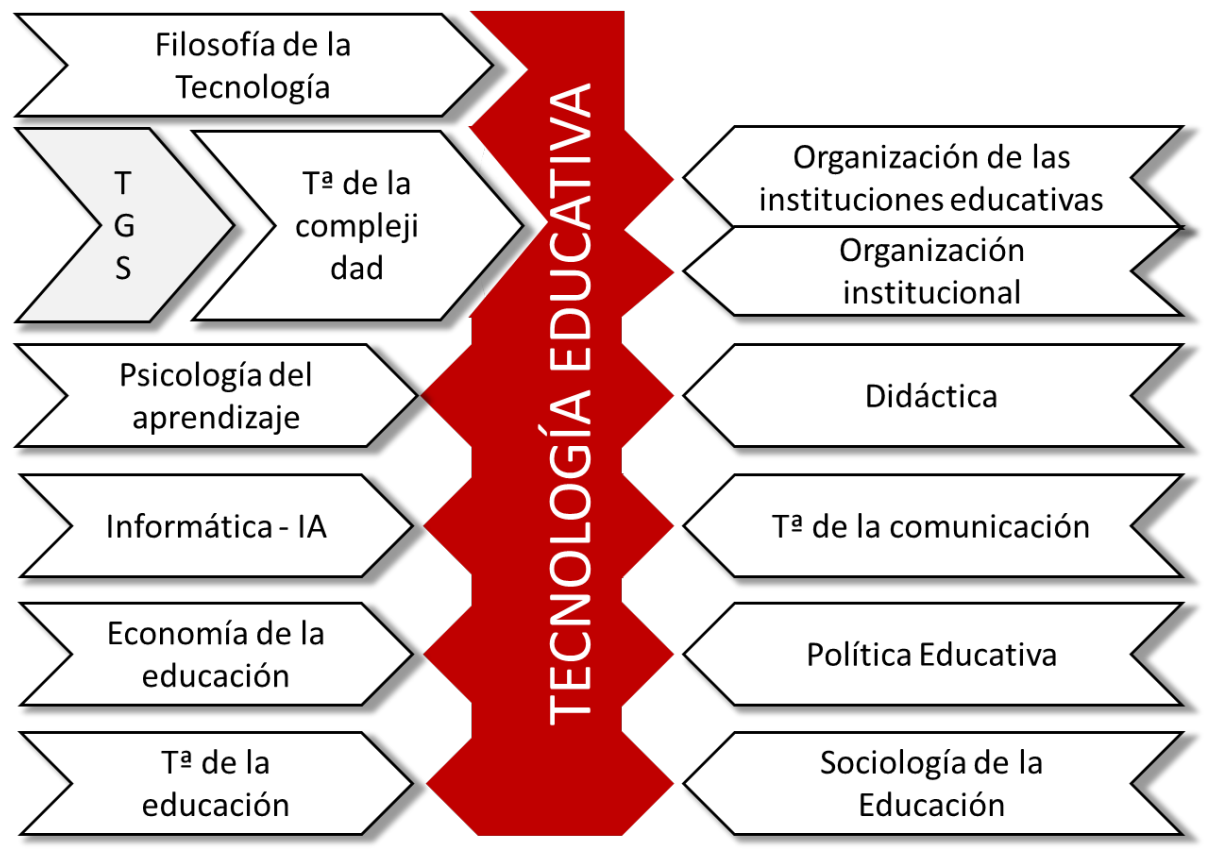

Figura 2. Soportes e interlocutores de la Tecnología Educativa contemporánea. Elaboración propia.

\section{V.La investigación en TE}

La tecnología educativa, como disciplina académica objeto de enseñanza e investigación, parece estar en medio de una tormenta perfecta. Una conjunción de factores internos y externos la está llevando hacia la más completa irrelevancia, una total ausencia de valor social, de capacidad y de legitimidad para orientar la acción educativa, para que los conocimientos que ha construido a lo largo del tiempo sean tenidos en cuenta a la hora de tomar decisiones y de orientar la práctica. En una época en la que la tecnología digital ha revolucionando todos los aspectos de las sociedades avanzadas, incluyendo la economía, la cultura, las relaciones interpersonales, la política, la ciencia, la información, etc., la investigación sobre la relación entre tecnología y educación no parece informar ni la práctica educativa en las aulas (nivel micro), ni la organización y gestión educativa (nivel meso), ni las decisiones de política educativa, como la dotación de infraestructuras, el currículum o la formación del profesorado (nivel macro) (McFarlane, 2019). Modas y la influencia de un poderoso mercado que disfraza el marketing de investigación dominan un panorama francamente preocupante en el que las grandes compañías de la tecnología se disputan el pastel de la inversión pública en tecnología (Edsurge, 2017) o invierten grandes sumas de dinero en convencer a la opinión pública, a los docentes y a los gestores políticos de la necesidad de orientar el currículum en la dirección que más les conviene (Player-Koro et al., 2017; Williamson, 2015).

Los análisis de la investigación empírica en TE de los últimos años no son precisamente complacientes. Nos la dibujan como metodológicamente pobre (Bulfin et al., 2014) y carente de ambición y compromiso teóricos (Bennett y Oliver, 2011; Bulfin, Henderson y Johnson, 2013; Jones y Czerniewicz, 2011; Markauskaite y Reimann, 2014). Bulfin et al. (2014) realizaron una encuesta sobre metodología de investigación a 462 investigadores académicos en tecnología educativa y medios educativos. Los resultados subrayan "una preferencia por formas relativamente básicas de investigación descriptiva, junto con una falta de capacidad en la recopilación y el análisis cuantitativos avanzados de datos" (p. 403). West \& Borup (2014), por ejemplo, en una revisión de la investigación sobre lo publicado en 10 revistas de "diseño instruccional y tecnología" señalaron la 
evidente orientación tecnocéntrica de la literatura académica, que puede deberse a una reacción al enorme desarrollo de las tecnologías de la computación, pero que "podría estar obstaculizando nuestra capacidad para tener influencia en el desarrollo proactivo de los sistemas, modelos, pedagogías, políticas y tecnologías que mejorarán el aprendizaje en el siglo XXI" (p. 553).

A diferencia de los cambios paradigmáticos seriales de ciencias como la física, descritos por Kuhn, Czerniewicz (2010) ha caracterizado los diferentes enfoques teóricos en la literatura sobre TE que coexisten en la actualidad como un campo de conocimiento "multiparadigmático", con "una estructura de conocimiento horizontal que consiste en "lenguajes" especializados con modos especializados de interrogación y criterios especializados para la construcción y circulación de textos. Estos "lenguajes" están formados por un cluster de elementos con criterios para textos legítimos, qué cuenta como evidencia y qué cuenta como preguntas legítimas" (p. 526). Así, Czerniewicz propone como "lenguajes" diferenciados a) el diseño instruccional, b) la learning technology o educational technology, c) la learning science, d) el computer-supported collaborative learning, e) el networked learning, y f) los enfoques posmodernos de la TE. ¿Son inconmensurables dichos lenguajes? ¿Son paradigmas diferentes y mutuamente excluyente o son diferentes perspectivas o enfoques sobre el mismo objeto y problemas comunes con voluntad de entendimiento?

Friesen (2009) ha señalado que la TE trata la construcción teórica y el trabajo empírico como actividades separadas. En base a Habermas, Friesen (2009) ha distinguido tres grandes orientaciones ne la investigación en TE: la instrumental (relacionada con intereses técnicos asociados con el trabajo o la producción), la práctica (relacionada con la interpretación o el significado) y la emancipadora (relacionada con la transformación de la educación). En un nivel más bajo, (Hannon \& Al-Mahmood, 2014) han afirmado que cuatro corrientes o tendencias dan forma a los actuales debates sobre el papel y la importancia de la teoría en TE:

1. La "práctica basada en la evidencia", que hace especial énfasis en "lo que funciona" en educación en general, pero sin preocuparse apenas en averiguar por qué y cómo funciona y que no suele tener en cuenta ni la complejidad de los contextos educativos ni los efectos colaterales de justificar con porcentajes la decisión política (Biesta, 2010; Davies, 1999; Greenhalgh y Russell, 2009; Zhao, 2017).

2. Una corriente, relativamente reciente, orientada al big data, las "analíticas del aprendizaje", cuyos defensores creen poder prescindir de teorías previas y esperan que algoritmos matemáticos encuentren patrones en los datos que revelen asociaciones entre variables que ayuden a describir el aprendizaje y a prescribir acciones pedagógicas (Jones \& Czerniewicz, 2011; Siemens \& Long, 2011).

3. La todavía fuerte influencia del diseño instruccional, una de las corrientes de la TE "clásica" anclada en un paradigma positivista y en perspectivas conductistas y cognitivistas del aprendizaje (Kirschner \& Kester, 2016; Spector, 2016).

4. La investigación basada en el diseño, orientada a la innovación educativa y a la formulación de principios generalizables de diseño de productos, procesos y procedimientos (Anderson \& Shattuck, 2012; De Benito \& Salinas, 2016; Zheng, 2015), aunque criticada porque "Ia teoría o incluso la crítica sigue notablemente ausente" (Bennett \& Oliver, 2011)

El escaso "músculo teórico" de la tecnología educativa se debe también, en gran parte, a las políticas editoriales de las revistas de mayor impacto, cuyo interés en publicar artículos teóricos es prácticamente inexistente. Una reciente revisión de los 503 artículos empíricos publicados en los últimos dos años en tres de las revistas de mayor impacto en la disciplina (Computers \& Education, Learning, Media and Technology y el British Journal of Educational Technology) concluye que más del $40 \%$ de los artículos analizados eran completamente a-teóricos (ninguna referencia a una teoría), el 25\% describió "vagamente" las teorías que usaron y "pocos artículos (77 de 503) informaron de hallazgos que nos ayuden a aprender algo nuevo sobre una teoría en particular" (Hew et al., 2019, p. 967). En otra revisión reciente, Reeves y Oh (2017) analizaron los objetivos y 
los métodos de los artículos publicados en dos periodos de seis años (de 1989 a 1994 y de 2009 a 2014) de la revista Educational Technology Research and Development, la principal publicación de la Association for Educational Communications and Technology (AECT). Entre otros aspectos, sus conclusiones destacan que la revista apenas ha publicado artículos desde perspectivas críticas o posmodernas, que utilicen metodologías cualitativas o basadas en el diseño y que la única forma de contribución teórica admitida parece ser la revisión de literatura. Si el objetivo, obligado, de los investigadores es publicar en revistas de alto impacto y los editores y revisores de éstas parece que rechazan sistemáticamente las contribuciones teóricas, no es extraño el pobre panorama que dibujan las revisiones sobre el avance de la teoría en la TE. Por otra parte, las corrientes que ven la TE como un campo de mera aplicación de otras disciplinas, especialmente la psicología y la teoría de los medios, y las que directamente se declaran "a-téoricas" y fían el avance del conocimiento al análisis de datos ignorando sus propias asunciones teóricas previas, contribuyen decisivamente al estado comatoso de la investigación en TE y, a la postre, a su irrelevancia práctica.

Si tenemos en cuenta que la investigación en TE es un campo de indagación, entonces es lógico que existan controversias (Reeves, 2000). Una de las disputas más obvias es entre aquellos que ven la TE como una rama de la ciencia o tecnología y aquellos que la ven más parecida a una artesanía o incluso a un arte. Otra controversia básica hace referencia a si debe realizarse investigación básica para construir teorías generalizables o perseguir la investigación aplicada para resolver problemas específicos (Salinas, 2012). Saeverot y Kvam (2019), han propuesto adoptar una perspectiva "estereoscópica" que combine las teorías prácticas y normativas, cuyo objetivo es mejorar la práctica educativa, con la teorías descriptivas y explicativas. Es más, diversos autores proponen la necesidad de aceptar cierto nivel de pluralismo metodológico, analítico y filosófico en la investigación en TE ya que "...brinda la oportunidad de una comprensión más profunda de preguntas de investigación complejas y múltiples perspectivas sobre la comprensión de datos" (Strydom \& Fourie-Malherbe, 2019, p. 189). Kimmons y Johnstun (2019, p. 10) reclaman pluralismo paradigmático en la formación de los investigadores en TE:

"...no solo estamos capacitando a positivistas, intérpretes, posmodernos, marxistas o feministas en nuestros programas y no solo evaluadores cualitativos, expertos en medidas cuantitativas, defensores de la justicia social o psicometristas, sino que también estamos capacitando a una nueva generación de profesionales que debe navegar el pluralismo a través de ciclos de crisis paradigmáticas personales."

Pero el pluralismo es una vía compleja. Como señalaron Yanchar y Williams (2006), los métodos que se utilizan en la investigación predeterminan la naturaleza de los resultados. Los supuestos subyacentes de un método sobre la naturaleza de la realidad, es decir, la ontología que subyace a toda epistemología, el contexto, el lenguaje, etc. "...constituyen colectivamente un mecanismo de investigación que produce o refleja descripciones y explicaciones no neutrales, sino interpretaciones enmarcadas en sus propios términos" ( $p .4)$. Si nos tomamos seriamente la teoría, entonces la formulación de las preguntas de investigación, el uso de unos métodos u otros y la interpretación de los resultados son deudoras de los marcos conceptuales que subyacen a métodos y prácticas de investigación. Para superar los dos extremos indeseados, el "eclecticismo metodológico" y la "rigidez paradigmática", dichos autores proponen prácticas de investigación que cumplan cinco criterios: que sean sensibles al contexto, creativas, conceptualmente conscientes, coherentes y abiertas a la crítica (p. 8-9).

Décadas de investigación experimental en TE con objetivos empíricos han proporcionado escasos fundamentos teóricos y principios para guiar la práctica educativa. Dada la pobre historia de otros enfoques metodológicos, Reeves (2000) señala que, si desde la TE se quiere contribuir significativamente a la reforma educativa, debe perseguir objetivos de desarrollo. La "ingeniería del aprendizaje" (véase Dede, Richards y Saxberg (2018), por ejemplo) orientada hacia la "resolución de problemas" (y "lo que funciona") ignora que un objetivo importante de la investigación es 
también detectar problemas y que la investigación educativa que opera en el modo de planteamiento de problemas en lugar del modo de resolución de problemas es en sí misma una forma de educación ya que trata de cambiar la mentalidad y las percepciones comunes, de exponer los supuestos y las condiciones de posibilidad de nuestras ideas, de entablar conversaciones continuas sobre qué es valioso en educación y en la sociedad en general... con el fin de mostrar que tal vez hay algo más que debería pedirse o buscarse (Biesta et al., 2019).

Una corriente con abundantes apoyos afirma que los desafíos y ámbitos de investigación y de práctica de diseño y desarrollo pueden ser abordados mediante el estudio académico de la enseñanza utilizando los métodos de la investigación basada en diseño. También se puede distinguir, a otro nivel de abstracción, una meta general común a todos los enfoques: reducir la incertidumbre en la toma de decisiones y en el diseño y desarrollo de intervenciones educativas. El término intervención sirve de denominador común para productos, programas, materiales, procedimientos, escenarios, procesos y otros similares. Esta meta general puede ser definida en dos objetivos más específicos que aplicar a los distintos enfoques en varios grados: a) proporcionar ideas (sugerencias, direcciones) para optimizar la calidad de la intervención a desarrollar; b) generar, articular y contrastar principios de diseño. Estos principios pueden ser de naturaleza substantiva, refiriéndose a las características de la intervención o de una naturaleza procedimental (cómo debe ser desarrollado). Los objetivos difieren en su contribución con relación a la 'práctica' o a la 'ciencia'. Mientras el primero (optimización de la intervención) está especialmente orientado hacia fines prácticos en una situación dada, el segundo (principios de diseño) refleja más fuertemente aspiraciones científicas o académicas, desde el momento en que está más explícitamente orientado hacia la producción de conocimiento de naturaleza generalizable.

Creemos que la investigación actual en TE debería tener en cuenta planteamientos de otras ciencias humanas y sociales que podrían enriquecer nuestra comprensión de la compleja relación entre tecnología y educación en los tiempos que vivimos. Uno de ellos, escasamente explorado, es el realismo crítico que intenta superar las limitaciones tanto de los enfoques positivistas como interpretativos (Archer, Bhaskar, Collier, Lawson y Norrie, 1998; Evans, 2011; Scott, 2005 y 2013). Otro enfoque prometedor es el sociomaterial (Decuypere \& Simons, 2016; Fenwick et al., 2015).

\section{VI.Conclusiones abiertas y debates consecuentes}

Como hemos afirmado en la introducción, el objetivo de este texto es una llamada a reflexión y al debate. Sin ánimo de "concluir", sino de "empezar" y con la mirada puesta en avanzar y proponer, queremos, llegados a este punto, hacer una breve síntesis de algunos de los puntos que creemos más importantes de todo lo planteado hasta ahora. El primero de ellos es nuestro punto de partida: la Tecnología Educativa es el campo de conocimiento que formula preguntas y busca respuestas de índole educativa acerca de la compleja relación entre las personas y la tecnología en todos los ámbitos de la educación. $Y$ desde esa perspectiva:

Es imprescindible utilizar en TE una conceptualización más amplia de la tecnología, que vaya más allá de su valor instrumental. De hecho, deberíamos hablar más de "tecnologías" que de "la tecnología".

Es imprescindible sobrepasar la visión reduccionista de la relación de la TE con la educación, siendo conscientes de que el impacto de la tecnología en la educación llega mucho más allá del diseño instruccional. La TE no solo se refiere a los recursos para usar en el aula. El Instructional Design es parte de la TE, pero no es toda la TE.

La TE se constituye como campo de estudio que debe mantener un diálogo profundo, fluido y 
constante con otras disciplinas de las ciencias de la educación para completarlas y completarse adecuadamente.

Si queremos aportar cuestiones relevantes a otros análisis o propuestas interdisciplinares -tan habituales por otra parte en nuestros días- es imprescindible tener claro qué y quiénes somos, y por lo mismo aportar algo de valor, sin ser fagocitados por otros planteamientos más claramente posicionados.

Parte de las dificultades de la TE quizá provengan de la irreductibilidad (y los problemas de comunicación) de las dos grandes tradiciones occidentales del estudio y la investigación de la educación: la anglosajona del currículum y la continental de la Pädagogik. El origen de la TE y el mercado actual de la TE impone conceptos cuya traducción es problemática y cuando creemos que hablamos de las mismas cosas en TE -aún cuando usamos a veces el mismo idioma- descubrimos que no es así.

Las propuestas de investigación tienen que contemplar tanto el avance de la teoría como la intervención efectiva en contextos reales, es decir, complejos. La TE no puede limitarse a la "crítica de la TE realmente existente" sino que debe elaborar propuestas de actuación en contextos educativos formales micro, meso y macro y no debe olvidar que la educación es mucho más que escuelas, aulas y evaluación de los aprendizajes, que el impacto de la tecnología nos llega desde múltiples ámbitos y está cambiando la manera en la que hacemos las cosas y, a la postre, quienes somos.

Finalmente, y quizá la convicción más firme de los autores, es que, en esta sociedad digital hipertecnificada (o post-digital), la agenda de la pedagogía no pueden establecerla las fuerzas del mercado o las fundaciones filantrópicas de las empresas tecnológicas: una TE crítica, comprometida, que haga avanzar la teoría y los principios y métodos de intervención educativa contextualizada, es más necesaria que nunca.

No son los únicos debates, sin duda. La crítica propositiva no puede ser una opción a estas alturas. Esperamos que el lector avance las suyas propias y emprendamos un camino de construcción -o reconstrucción- que nos permita avanzar desde este supuesto punto de irrelevancia, hacia las posiciones que se necesita.

\section{Referencias}

Achterhuis, H. (Ed.). (2001). American Philosophy of Technology: The Empirical Turn. Indiana University Press. http://www.journals.uchicago.edu/doi/10.1086/374173

Adell, J. (2010). Educación 2.0. En C. Barba \& S. Capella (Eds.), Ordenadores en las aulas. La clave es la metodología. (pp. 19-33). Graó.

Adell, J. (2018). Más allá del instrumentalismo en tecnología educativa. En J. Gimeno (Ed.), Cambiar los contenidos, cambiar la educación. Morata.

AECT Definition and Terminology Committee. (2004). The Meanings of Educational Technology. AECT Definition and Terminology Committee document \#MM4.0. University of Indiana. https://www.tlu.ee/ kpata/haridustehnoloogiaTLU/defineeducationaltechnology.pdf

AECT Task Force on Definition and Terminology (Ed.). (1979). The definition of educational technology. Association for Educational Communications and Technology.

Anderson, T., \& Shattuck, J. (2012). Design-Based Research: A Decade of Progress in Education Research? Educational Researcher, 41(1), 16-25. https://doi.org/10.3102/0013189X11428813

Archer, M. S., Bhaskar, R., Collier, A., Lawson, T., \& Norrie, A. (Eds.). (1998). Critical realism: Essential 
readings. Routledge.

Area, M. (1991a). La tecnología educativa en la actualidad: Las evidencias de una crisis. Qurriculum. Revista de Teoría, Investigación y Práctica Educativa, 3, 11. http://www.quadernsdigitals.net/index.php?accionMenu=hemeroteca.VisualizaArticuloIU.visualiza\&arti culo_id $=2650$

Area, M. (1991b). Los medios, los profesores y el currículo. Sendai.

Area, M. (2004). Los medios y las tecnologías en la educación (Edición: edición). Pirámide.

Area, M., Gros, B., \& Marzal, M. (2008). Alfabetizaciones y tecnologías de la información y la comunicación (Edición: 1). Sintesis.

Attwell, G. (2019). Personal Learning Environments. En Literacias críticas e boas práticas mediáticas. Coleção Educação, Centro de Estudos de Comunicação e Sociedade, Universidade do Minho.

Azevedo, R. (2009). Theoretical, conceptual, methodological, and instructional issues in research on metacognition and self-regulated learning: A discussion. Metacognition and Learning, 4(1), 87-95. Scopus. https://doi.org/10.1007/s11409-009-9035-7

Baker, T., Smith, L., \& Anissa, N. (2019). Educ-AI-tion Rebooted? Exploring the future of artificial intelligence in schools and colleges. NESTA. https://www.nesta.org.uk/report/education-rebooted/

Ball, S. (1995). Intellectuals or technicians? The urgent role of theory in educational studies. British Journal of Educational Studies, 43(3), 255-271. https://doi.org/10.1080/00071005.1995.9974036

Ball, S. (2013). The education debate. Policy Press.

Ball, S. (2016). Following policy: Networks, network ethnography and education policy mobilities. Journal of Education Policy, 31(5), 549-566. https://doi.org/10.1080/02680939.2015.1122232

Ball, S., Maguire, M., Braun, A., Perryman, J., \& Hoskins, K. (2011). Assessment technologies in schools: Deliverology and the play of dominations. Research Papers in Education, 27(5), 513-533. https://doi.org/10.1080/02671522.2010.550012

Bartolomé, A. (1988). Tecnología Educativa. Proyecto docente presentado en el concurso de acceso a la plaza de Profesor Titular de Universidad (Universidad de Barcelona). http://www.Imi.ub.es/personal/bartolome/articuloshtml/bartolome_tit_88/\#capitol5

Bartolomé, A. (2008). Vídeo digital y educación (Edición: 1). Editorial Síntesis, S. A.

Bartolomé, A., Castañeda, L., \& Adell, J. (2018). Personalisation in educational technology: The absence of underlying pedagogies. International Journal of Educational Technology in Higher Education, 15(1), 14. https://doi.org/10.1186/s41239-018-0095-0

Beer, D. (2017). The social power of algorithms. Information, Communication \& Society, 20(1), 1-13. https://doi.org/10.1080/1369118X.2016.1216147

Bennett, S., \& Oliver, M. (2011). Talking back to theory: The missed opportunities in learning technology research. Research in Learning Technology, 199-189. https://doi.org/10.1080/21567069.2011.624997

Biesta, G. J. J. (2010). Why 'What Works' Still Won't Work: From Evidence-Based Education to Value-Based Education. Studies in Philosophy and Education, 29, 491-503. https://doi.org/10.1007/s11217-0109191-x

Biesta, G. J. J. (2011). Disciplines and theory in the academic study of education: A comparative analysis of the Anglo-American and Continental construction of the field. Pedagogy, Culture \&amp; Society, 19, 175192. https://doi.org/10.1080/14681366.2011.582255

Biesta, G., Filippakou, O., Wainwright, E., \& Aldridge, D. (2019). Why educational research should not just solve problems, but should cause them as well. British Educational Research Journal, 45(1), 1-4. https://doi.org/10.1002/berj.3509

Blumer, H. (1962). Symbolic Interactionism: Perspective and Method. University of California Press. 
Bower, M. (2019). Technology-mediated learning theory. British Journal of Educational Technology, 50(3), 1035-1048. https://doi.org/10.1111/bjet.12771

Brey, P. (2010). Philosophy of Technology after the Empirical Turn. Techné: Research in Philosophy and Technology, 14(1), 36-48.

Bromley, H. (1997). The Social Chicken and The Technological Egg: Educational Computing and the Technology/Society Divide. Educational Theory, 47(1), 51-65. https://doi.org/10.1111/j.17415446.1997.00051.x

Buckingham, D. (2006). Defining digital literacy-What do young people need to know about digital media? Digital Kompetanse, 1(Jubileumsnummer), 263-276.

Buckingham, D., Scanlon, M., \& Sefton-Green, J. (2001). Selling the Digital Dream. Marketing educational technology to teachers and parents. En A. M. Loveless \& V. Ellis (Eds.), ICT, Pedagogy and the Curriculum: Subject to Change. Routledge.

Bulfin, S., Henderson, M., Johnson, N. F., \& Selwyn, N. (2014). Methodological capacity within the field of "educational technology" research: An initial investigation. $\mathrm{Br} J$ Educ Technol, 45, 403-414. https://doi.org/10.1111/bjet.12145

Byrne, D. (2005). Complexity, Configurations and Cases. Theory, Culture \& Society, 22(5), 95-111. https://doi.org/10.1177/0263276405057194

Cabero, J. (1996). Nuevas tecnologías, comunicación y educación. Edutec. Revista Electrónica de Tecnología Educativa, O(1), 001. https://doi.org/10.21556/edutec.1996.1.576

Cabero, J. (Ed.). (2007). Tecnología Educativa (Edición: 1). McGraw-Hill Interamericana de España S.L.

Cabero, J., \& Martínez Sánchez, F. (Eds.). (1995). Nuevos canales de comunicacion en la enseñanza. Editorial Universitaria Ramón Areces.

Caena, F. (2013). Supporting teacher competence development: For better learning outcomes. European Commission. Education and Training. http://ec.europa.eu/dgs/education_culture/repository/education/policy/school/doc/teachercomp_en.pd f

Carlson, J., \& Johnston, L. (2015). Data information literacy: Librarians, data, and the education of a new generation of researchers.

Castañeda, L. (2016). Suelo y sol: Más pensamiento y contexto para la innovación educativa. Boletín de la Institución Libre de Enseñanza, 104, 37-50.

Castañeda, L. (2019). Debates sobre Tecnología y Educación: Caminos contemporáneos y conversaciones pendientes. RIED. Revista Iberoamericana de Educación a Distancia, 22(1). https://doi.org/10.5944/ried.22.1.23020

Castañeda, L., Esteve, F., \& Adell, J. (2018). ¿Por qué es necesario repensar la competencia docente para el mundo digital? RED. Revista de Educación a Distancia, 56. https://doi.org/10.6018/red/56/6

Castañeda, L., \& Selwyn, N. (2018). More than tools? Making sense of the ongoing digitizations of higher education. International Journal of Educational Technology in Higher Education, 15, 22. https://doi.org/10.1186/s41239-018-0109-y

Clark, A. (2003). Natural-Born Cyborgs: Minds, Technologies, and the Future of Human Intelligence. Oxford University Press.

Clark, R. E. (1994). Media will never influence learning. Educational Technology Research and Development, 42(2), 21-29. https://doi.org/10.1007/BF02299088

Clow, D. (2012). The Learning Analytics Cycle: Closing the Loop Effectively. Proceedings of the 2Nd International Conference on Learning Analytics and Knowledge, 134-138. https://doi.org/10.1145/2330601.2330636

Cobo, C. (2016). La Innovación Pendiente. Colección Fundación Ceibal/ Debate. http://innovacionpendiente.com/ 
Colebatch, H. K. (2015). Participation and Professionalism in Policy Work: Questions for Polities in Transition. Anali Hrvatskog politolo|textbackslashvskog dru|textbackslashvstva, 11(1), 45-54.

Coll, C. (2016). La personalización del aprendizaje escolar El qué, el por qué y el cómo de un reto insoslayable. En J. Vilalta (Ed.), Reptes de l'educació a Catalunya. Anuari d'Educació 2015 (pp. 43-104). Fundació Jaume Bofill. https://www.researchgate.net/publication/305999815_La_personalizacion_del_aprendizaje_escolar_El _que_el_por_que_y_el_como_de_un_reto_insoslayable

Costa, C., Hammond, M., \& Younie, S. (2019). Theorising technology in education: An introduction. Technology, Pedagogy and Education, 28(4), 395-399. https://doi.org/10.1080/1475939X.2019.1660089

Cuban, L. (1993). Computers meet classroom: Classroom wins. Teachers College Record, 95(2), 185-210.

Cuban, L. (2009). Oversold and underused. Harvard university press.

Czerniewicz, L. (2008). Distinguishing the Field of Educational Technology. Electronic Journal of e-Learning, $6(3), 171-178$.

Czerniewicz, L. (2010). Educational technology-Mapping the terrain with Bernstein as cartographer. Journal of Computer Assisted Learning, 26, 523-534.

Da Silva, T. T. (1999). Documentos de identidade: Uma introdução às teorias do currículo. Autêntica Editora.

Dabbagh, N., \& Kitsantas, A. (2012). Personal Learning Environments, social media, and self-regulated learning: A natural formula for connecting formal and informal learning. The Internet and Higher Education, 15(1), 3-8. https://doi.org/10.1016/j.iheduc.2011.06.002

Davies, P. (1999). What is evidence-based education? British journal of educational studies, 47(2), $108-121$.

De Benito, B., \& Salinas, J. M. (2016). La Investigación Basada en Diseño en Tecnología Educativa. Revista Interuniversitaria de Investigación en Tecnología Educativa. https://doi.org/10.6018/riite2016/260631

de Laat, M., \& Dohn, N. B. (2019). Is Networked Learning Postdigital Education? Postdigital Science and Education, 1(1), 17-20. https://doi.org/10.1007/s42438-019-00034-1

de Laat, P. B. (2017). Algorithmic Decision-Making Based on Machine Learning from Big Data: Can Transparency Restore Accountability? Philosophy \& Technology. https://doi.org/10.1007/s13347-0170293-z

de Pablos, J. (2009). Tecnología educativa: La formación del profesorado en la era de Internet (Edición: 1). Ediciones Aljibe, S.L.

de Vries, M. (2012). Philosophy of Technology. En P. J. Williams (Ed.), Technology Education for Teachers (pp. 15-34). Sense Publishers.

Decuypere, M., \& Simons, M. (2016). Sobre el potencial crítico de los enfoques sociomateriales en educación. Teoría de la Educación. Revista Interuniversitaria, 28, 25. https://doi.org/10.14201/teoredu20162812544

Dede, C., Richards, J., \& Saxberg, B. (2018). Learning Engineering for Online Education: Theoretical Contexts and Design-based Examples. Routledge.

Dhar, V. (2013). Data Science and Prediction. Commun. ACM, 56(12), 64-73. https://doi.org/10.1145/2500499

Edsurge. (2017). The State of Edtech 2016. Edsurge. https://www.edsurge.com/research/reports/the-state-ofedtech-2016

Ely, D. (2008). Frameworks of educational technology. British Journal of Educational Technology, 39(2), 244250. https://doi.org/10.1111/j.1467-8535.2008.00810.x

Eraut, M. (1989). Conceptual frameworks and historial development. En M. Eraut (Ed.), International Encyclopedia of Educational Technology (1 edition, pp. 11-21). Pergamon.

Esteve, F., Castañeda, L., \& Adell, J. (2018). Un modelo holístico de competencia docente para el mundo digital. Revista Interuniversitaria de Formación del Profesorado, 32(1).

European Union. (2015a, noviembre 13). DigCompOrg Framework-EU Science Hub-European Commission. EU 
Science Hub. https://ec.europa.eu/jrc/en/digcomporg/framework

European Union. (2015b, diciembre 11). Promoting Effective Digital-Age Learning: A European Framework for Digitally-Competent Educational Organisations - EU Science Hub - European Commission. EU Science Hub. https://ec.europa.eu/jrc/en/publication/eur-scientific-and-technical-research-reports/promotingeffective-digital-age-learning-european-framework-digitally-competent-educational

Evans, M. A. (2011). A critical-realist response to the postmodern agenda in instructional design and technology: A way forward. Educational Technology Research and Development, 59(6), 799-815. https://doi.org/10.1007/s11423-011-9194-5

Feenberg, A. (2017). Technosystem: The Social Life of Reason. Harvard University Press.

Fenwick, T., Edwards, R., \& Sawchuk, P. (2011). Emerging Approaches to Educational Research: Tracing the Socio-Material ( 1 edition). Routledge.

Ferres, J. (2014). Las pantallas y el cerebro emocional. Gedisa. https://www.amazon.es/pantallas-cerebroemocional-Comunicaci\%C3\%B3n-Educativa-ebook/dp/B0168HL8HW/ref=sr_1_1?s=digitaltext\&ie $=$ UTF8\&qid $=1523665036 \& s r=1-1 \&$ keywords $=$ joan + ferres + pantallas

Floridi, L. (Ed.). (2016). The Routledge Handbook of Philosophy of Information (1. a ed.). Routledge. https://doi.org/10.4324/9781315757544

Floridi, L. (2017). The Fourth Revolution: How the Infosphere Is Reshaping Human Reality. Oxford University Press.

Friesen, N. (2009). Re-thinking e-learning research: Foundations, methods, and practices (Vol. 333). Peter Lang.

Glister, P., Rlister, P., \& Gilster, P. (1998). Digital Literacy (Edición: New Ed). John Wiley \& Sons, Inc.

Granovetter, M. (1983). The Strength of Weak Ties: A Network Theory Revisited. Sociological Theory, 1, 201233. https://doi.org/10.2307/202051

Hamilton, E., \& Friesen, N. (2013). Online Education: A Science and Technology Studies Perspective / Éducation en ligne: Perspective des études en science et technologie. Canadian Journal of Learning and Technology / La Revue Canadienne de l'apprentissage et de La Technologie, 39(2). https://doi.org/10.21432/T2001C

Hannon, J., \& Al-Mahmood, R. (2014). The place of theory in educational technology research. Rhetoric and reality: Critical perspectives on educational technology: Proceedings of the 31st Annual ASCILITE Conference, 745-750.

Hayles, N. K. (2007). Hyper and Deep Attention: The Generational Divide in Cognitive Modes. Profession, 2007(1), 187-199. https://doi.org/10.1632/prof.2007.2007.1.187

Hayles, N. K. (2012). How we think: Digital media and contemporary technogenesis. The University of Chicago Press.

Hayles, N. K. (2016). Cognitive assemblages: Technical agency and human interactions. Critical Inquiry, 43(1), 32-55.

Hew, K. F., Lan, M., Tang, Y., Jia, C., \& Lo, C. K. (2019). Where is the "theory" within the field of educational technology research? British Journal of Educational Technology, 50(3), 956-971. https://doi.org/10.1111/bjet. 12770

Honan, E. (2010). Mapping discourses in teachers' talk about using digital texts in classrooms. Discourse: Studies in the Cultural Politics of Education, 31(2), 179-193. https://doi.org/10.1080/01596301003679701

Ibrahim, A. A. (2015). Evolutionary Nature of the Definition of Educational Technology. International Journal of Social Sciences \& Education, 5(2), 233-239.

Jameson, J. (2019). Developing critical and theoretical approaches to educational technology research and practice. British Journal of Educational Technology, 50(3), 951-955. https://doi.org/10.1111/bjet. 12775 
Januszewski, A. (2001). Educational technology: The development of a concept. Libraries Unlimited.

Jones, C., \& Czerniewicz, L. (2011). Theory in learning technology. Research in Learning Technology, 19, 173177. https://doi.org/10.1080/21567069.2011.632491

Jones, C., \& Kennedy, G. (2011). Stepping beyond the paradigm wars: Pluralist methods for research in learning technology. Research in Learning Technology, 19(sup1), 7798. https://doi.org/10.3402/rlt.v19s1/7798

Kimmons, R., \& Johnstun, K. (2019). Navigating Paradigms in Educational Technology. TechTrends, 63(5), 631641. https://doi.org/10.1007/s11528-019-00407-0

Kirschner, P. A., \& Kester, L. (2016). Towards a Research Agenda for Educational Technology Research. En N. Rushby \& D. W. Surry (Eds.), The Wiley Handbook of Learning Technology (pp. 523-541). John Wiley \& Sons, Inc. https://doi.org/10.1002/9781118736494.ch27

Knight, S., Allen, L., Gibson, A., McNamara, D., \& Buckingham Shum, S. (2017). Writing analytics literacy: Bridging from research to practice. 496-497. https://doi.org/10.1145/3027385.3029425

Kroes, P., \& Verbeek, P.-P. (Eds.). (2014). The Moral Status of Technical Artefacts (Vol. 17). Springer Netherlands. https://doi.org/10.1007/978-94-007-7914-3

Lai, J. W. M., \& Bower, M. (2019). How is the use of technology in education evaluated? A systematic review. Computers \& Education, 133, 27-42. https://doi.org/10.1016/j.compedu.2019.01.010

Liyanagunawardena, T. R., Adams, A. A., \& Williams, S. A. (2013). MooCs: A Systematic Study of the Published Literature 2008-2012. International Review of Research in Open and Distance Learning, 14(3), 202-227. https://doi.org/10.19173/irrodl.v14i3.1455

Martínez Bonafé, J. (2001). Arqueología del concepto «compromiso social» en el discurso pedagógico y de formación docente. Revista Electrónica de Investigación Educativa (REDIE), 3(1). http://www.redalyc.org/articulo.oa?id=15503106

Martínez, F., \& Prendes, M. P. (2003). ¿Adónde va la educación en un mundo de tecnologías? En F. Martínez (Ed.), Redes de comunicación en la enseñanza (pp. 281-300). Paidós. https://dialnet.unirioja.es/servlet/articulo?codigo $=756021$

Martínez, F. (2007). La integración escolar de las nuevas tecnologías. En J. Cabero (Ed.), Nuevas Tecnologías aplicadas a la educación (pp. 21-40). McGraw-Hill Interamericana de España S.L.

Mayer, R. E. (2019). Computer Games in Education. Annual Review of Psychology, 70(1), 531-549. https://doi.org/10.1146/annurev-psych-010418-102744

McCann, E. (2011). Urban Policy Mobilities and Global Circuits of Knowledge: Toward a Research Agenda. Annals of the Association of American Geographers, 101(1), 107-130. https://doi.org/10.1080/00045608.2010.520219

McFarlane, A. E. (2019). Devices and desires: Competing visions of a good education in the digital age. British Journal of Educational Technology, 50(3), 1125-1136. https://doi.org/10.1111/bjet.12764

Menashy, F. (2015). Understanding the roles of non-state actors in global governance: Evidence from the Global Partnership for Education. Journal of Education Policy, 31(1), 98-118. https://doi.org/10.1080/02680939.2015.1093176

Mintrom, M., \& Vergari, S. (1998). Policy Networks and Innovation Diffusion: The Case of State Education Reforms. The Journal of Politics, 60(1), 126-148. https://doi.org/10.2307/2648004

Moreno, G. A., Jimenez, J. A., \& Puche, W. S. (2016). Ubiquitous Personal Learning Environment model (uPLEMO). 2016 Technologies Applied to Electronics Teaching (TAEE), 1-8. https://doi.org/10.1109/TAEE.2016.7528383

Nichols, R. S., \& Allen-Brown, V. (1996). Critical Theory and Education Technology. En D. H. Jonassen (Ed.), Handbook of research for educational communications and technology (pp. 226-252). Simon \& Schuster.

Oliver, M. (2011). Technological determinism in educational technology research: Some alternative ways of 
thinking about the relationship between learning and technology: Educational technology and determinism. Journal of Computer Assisted Learning, 27(5), 373-384. https://doi.org/10.1111/j.13652729.2011.00406.x

Oliver, M. (2016). What is Technology. En N. Rushby \& D. Surry (Eds.), Wiley Handbook of Learning Technology (Edición: 1). Wiley Handbooks in Education.

Pangrazio, L., \& Selwyn, N. (2018). 'Personal data literacies': A critical literacies approach to enhancing understandings of personal digital data. New Media \& Society, 1461444818799523. https://doi.org/10.1177/1461444818799523

Papert, S. (1981). Computers and computer cultures. Creative Computing, 7(3), 82-92.

Parejo, N., \& Olmedo, E. M. (2017). ANÁLISIS DEL PLE Y OLE DE UN GRUPO DE INVESTIGACIÓN DE LA UNIVERSIDAD DE GRANADA. UN ESTUDIO DE CASOS CON CANVAS. 6.

Player-Koro, C., Bergviken Rensfeldt, A., \& Selwyn, N. (2017). Selling tech to teachers: Education trade shows as policy events. Journal of Education Policy, 1-22. https://doi.org/10.1080/02680939.2017.1380232

Prendes, M. P. (1997). Tecnología Educativa. Proyecto docente presentado en el concurso de acceso a la plaza de Profesor Titular de Universidad (Universidad de Murcia).

Provost, F., \& Fawcett, T. (2013). Data Science and its Relationship to Big Data and Data-Driven Decision Making. Big Data, 1(1), 51-59. https://doi.org/10.1089/big.2013.1508

Reeves, T. C., \& Oh, E. G. (2017). The goals and methods of educational technology research over a quarter century (1989-2014). Educational Technology Research and Development, 65(2), 325-339.

Reiser, R. A. (2001). A History of Instructional Design and Technology: Part I: A History of Instructional Media. Educational Technology Research and Development, 49(1), 53-64. http://www.jstor.org/stable/30220299

Rodríguez, J., \& Martínez-Bonafé, J. (2016). Libros de texto y control del curriculum en el contexto de la sociedad digital. Cadernos CEDES, 36(100), 319-336. https://doi.org/10.1590/cc010132622016171317

Rogers, E. M. (2003). Diffusion of Innovations, 5th Edition (Edición: 5th Edition.). Simon \& Schuster.

Saettler, P. (1978). The Roots of Educational Technology. Programmed Learning and Educational Technology, 15(1), 7-15. https://doi.org/10.1080/0033039780150102

Saettler, P. (1990). Evolution of American Educational Technology (Edición: Revised). Libraries Unlimited Inc.

Saeverot, H., \& Kvam, V. (2019). An alternative model of researching educational practice: A pedagogicstereoscopic point of view. British Educational Research Journal, 45(1), 201-218. https://doi.org/10.1002/berj.3493

Salinas, J. (1991). Proyecto docente presentado en el concurso de acceso a la plaza de Profesor Titular de Universidad del área de Didáctica y Organización Escolar (Universitat de les Illes Balears).

Sancho, J. M. (Ed.). (1994). Para una tecnología educativa. Horsori.

Sarramona, F., \& Fernández, A. (1978). La educación. Constantes y problemática actual. CEAC, Barcelona.

Scott, D. (2005). Critical realism and empirical research methods in education. Journal of Philosophy of Education, 39(4), 633-646. Scopus. https://doi.org/10.1111/j.1467-9752.2005.00460.x

Scott, David. (2013). Education, Epistemology and Critical Realism (1. ${ }^{\mathrm{a}}$ ed.). Routledge. https://doi.org/10.4324/9780203883099

Selwyn, N. (2016a). Is technology good for education? Polity Press.

Selwyn, N. (2016b). ¿Nuevas culturas del aprendizaje ?: (Una conversación con Linda Castañeda). Boletín de la Institución Libre de Enseñanza, 104, 51-77.

Selwyn, N. (2017). Education and technology: Critical questions. En G. Ferreira, L. Da Silva Rosado, \& J. Sá Carvalho (Eds.), Education and Technology: Critical approaches (pp. 105-122). SESES. https://osf.io/preprints/socarxiv/rmyg8/ 
Shannon, C., \& Weaver, W. (1949). The Mathematical Theory of Communication. The University of Illinois Press.

Siemens, G., \& Long, P. (2011). Penetrating the fog: Analytics in learning and education. EDUCAUSE review, $46(5), 30$.

Spector, J. M. (2016). Foundations of educational technology: Integrative approaches and interdisciplinary perspectives. Routledge. Taylor \& Francis Group.

Spring, J. (2012). Globalization of Education. International Journal of Chinese Education, 1(2), 139-176. https://doi.org/10.1163/22125868-12340002

Starkey, L. (2012). Teaching and Learning in the Digital Age (Edición: 1). Routledge.

Stiegler, B. (2015). States of shock: Stupidity and knowledge in the twenty-first century. Polity.

Stone, D. (2008). Global Public Policy, Transnational Policy Communities, and Their Networks. Policy Studies Journal, 36(1), 19-38. https://doi.org/10.1111/j.1541-0072.2007.00251.x

Strydom, S., \& Fourie-Malherbe, M. (2019). Pluralism as a Vehicle for Theory-Building in Educational Technology Research. En J. Huisman \& M. Tight (Eds.), Theory and Method in Higher Education Research (Vol. 5, pp. 173-191). Emerald.

Swan, M. (2013). The Quantified Self: Fundamental Disruption in Big Data Science and Biological Discovery. Big Data, 1(2), 85-99. https://doi.org/10.1089/big.2012.0002

Taffel, S. (2016). Perspectives on the postdigital: Beyond rhetorics of progress and novelty. Convergence, 22(3), 324-338. https://doi.org/10.1177/1354856514567827

Tamim, R. M., Bernard, R. M., Borokhovski, E., Abrami, P. C., \& Schmid, R. F. (2011). What Forty Years of Research Says About the Impact of Technology on Learning: A Second-Order Meta-Analysis and Validation Study. Review of Educational Research, 81(1), 4-28. https://doi.org/10.3102/0034654310393361

Thomson, P., Hall, C., \& Jones, K. (2010). Maggies day: A smallscale analysis of English education policy. Journal of Education Policy, 25(5), 639-656. https://doi.org/10.1080/02680931003783346

Trisha Greenhalgh, \& Jill Russell. (2009). Evidence-Based Policymaking: A Critique. Perspectives in Biology and Medicine, 52(2), 304-318. https://doi.org/10.1353/pbm.0.0085

Vázquez-Cano, E., \& Meneses, E. L. (2015). La filosofía educativa de los MOOC y la educación universitaria. RIED. Revista Iberoamericana de Educación a Distancia, 18(2), 25-37. https://doi.org/10.5944/ried.18.2.14261

Veletsianos, G., \& Moe, R. (2017, abril). The Rise of Educational Technology as a Sociocultural and Ideological Phenomenon. Educause Review. https://er.educause.edu/articles/2017/4/the-rise-of-educationaltechnology-as-a-sociocultural-and-ideological-phenomenon

Waller, M. A., \& Fawcett, S. E. (2013). Data Science, Predictive Analytics, and Big Data: A Revolution That Will Transform Supply Chain Design and Management. Journal of Business Logistics, 34(2), 77-84. https://doi.org/10.1111/jbl.12010

Watters, A. (2016). The Curse of the Monsters of Education Technology. Amazon.

Webster, M. D. (2017). Philosophy of Technology Assumptions in Educational Technology Leadership. Educational Technology \& Society, 20(1), 25-36.

West, R. E., \& Borup, J. (2014). An analysis of a decade of research in 10 instructional design and technology journals: Analysis of IDT research. British Journal of Educational Technology, 45(4), 545-556. https://doi.org/10.1111/bjet.12081

Whitson, J. R. (2013). Gaming the quantified self. Surveillance and Society, 11(1-2), 163-176. Scopus.

Williamson, B. (2013). The future of the curriculum: School knowledge in the digital age. MIT Press.

Williamson, B. (2015). Political computational thinking: Policy networks, digital governance and learning to code. Critical Policy Studies, 10(1), 39-58. https://doi.org/10.1080/19460171.2015.1052003 
Williamson, B. (2017). Moulding student emotions through computational psychology: Affective learning technologies and algorithmic governance. Educational Media International, 1-22. https://doi.org/10.1080/09523987.2017.1407080

Williamson, B., Potter, J., \& Eynon, R. (2019). New research problems and agendas in learning, media and technology: The editors' wishlist. Learning, Media and Technology, 44(2), 87-91. https://doi.org/10.1080/17439884.2019.1614953

Winner, L. (1980). Do Artifacts Have Politics? Daedalus, 109(1), 121-136.

Yanchar, S. C., \& Williams, D. D. (2006). Reconsidering the compatibility thesis and eclecticism: Five proposed guidelines for method use. Educational researcher, 35(9), 3-12.

Zawacki-Richter, O., Marín, V. I., Bond, M., \& Gouverneur, F. (2019). Systematic review of research on artificial intelligence applications in higher education - where are the educators? International Journal of Educational Technology in Higher Education, 16(1), 39. https://doi.org/10.1186/s41239-019-0171-0

Zhao, Y. (2017). What works may hurt: Side effects in education. Journal of Educational Change, 18(1), 1-19. https://doi.org/10.1007/s10833-016-9294-4

Zheng, L. (2015). A systematic literature review of design-based research from 2004 to 2013. Journal of Computers in Education, 2(4), 399-420. https://doi.org/10.1007/s40692-015-0036-z 